\title{
THE JUMP COMPONENT OF THE VOLATILITY STRUCTURE OF INTEREST RATE FUTURES MARKETS: AN INTERNATIONAL COMPARISON
}

\author{
CARL CHIARELLA* AND THUY-DUONG TÔ** \\ School of Finance and Economics \\ University of Technology, Sydney \\ PO Box 123 \\ Broadway NSW 2007 \\ Australia \\ *Carl.Chiarella@uts.edu.au \\ **Thuy.D.To@uts.edu.au
}

\begin{abstract}
We propose a generalization of the Shirakawa (1991) model to capture the jump component in fixed income markets. The model is formulated under the Heath, Jarrow and Morton (1992) framework, and allows the presence of a Wiener noise and a finite number of Poisson noises, each associated with a time deterministic volatility function. We derive the evolution of the futures price and use this evolution to estimate the model parameters via the likelihood transformation technique of Duan (1994). We apply the method to the short term futures contracts traded on CME, SFE, LIFFE and TIFFE, and find that each market is characterized by very different behaviour.
\end{abstract}

Key words: Term structure; Heath-Jarrow-Morton; Jump-diffusion; FIML; Likelihood transformation; Interest rate futures;

JEL classifications: C51, E43, G12, G13

Date: Current revision: Jan 6, 2003.

** Corresponding author

The authors would like to thank helpful comments from participants at the 2002 Quantitative Method in Finance Conference and the 15th Australasian Finance and Banking Conference. All remaining errors are our own responsibility. 


\section{INTRODUCTION}

The financial market is inevitably affected by information arrivals. As argued in Merton (1976) in relation to the jump-diffusion stock market model, the release of routine trading information will result in smooth changes in the underlying stock return process whereas bursts of information are often reflected in price behaviour as discontinuous jumps. The existence of jump components has been documented in the literature for the stock market and the exchange rate market, for example in Ball and Torous (1983, 1985), Jorion (1988), and recently in Bates (1996, 2000), Jiang (1999) and Pan (2002).

The fixed-income market is equally substantially impacted by information surprises. As cited in Das (2002), a number of researchers have found that economic news announcements and other releases of information have an impact on the Treasury bond market. Such findings include those of Hardouvelis (1988), Dwyer and Hafer (1989) and more recently, Balduzzi, Green and Elton (1998) and Green (1998). Therefore, jump-diffusion models for interest rates are proposed and analyzed in Babbs and Webber (1995), Naik and Lee (1995), El-Jahel, Lindberg and Perraudin (1997), Piazzesi (1999) and Das (2002), albeit the specification of the jump models are different. The general models of Chacko (1998), Chacko and Das (2002) and Singleton (2001) also contain jumps.

The above-mentioned works have extensively focused on the spot rate of interest. In this paper, we study jump-diffusion interest rate models under the general framework of Heath, Jarrow and Morton (1992) (hereafter HJM), which starts with specification of the dynamics of the instantaneous forward rate, under which the spot rate is one particular rate, ie. the instantaneous forward rate with zero maturity. The HJM model is an arbitrage-free model which matches the current term structure by construction, and relies on no assumption about investor preferences. The model offers a parsimonious representation of the market dynamics and requires only specification of the form of the forward rate volatility function.

The HJM model in the form of a diffusion process is difficult to estimate due to its (in general) non-Markovian nature. When jumps are introduced into the model, the degree of complexity is magnified. In this paper, we only consider time-deterministic diffusion volatility processes. Unlike previous studies on jump diffusion of the spot 
rate of interest, where the jump size is assumed to be drawn from a continuous distribution, we use multiple jump processes, each of which is associated with a constant jump value scaled by a time-deterministic jump volatility. The advantage of this approach is that if the jump size is distributed with an infinite number of possible realizations (as in the case of a continuous distribution such as the normal distribution), under the HJM framework, the market will not be complete (see Björk, Kabanov and Runggaldier (1997) for a detailed discussion of this issue), and therefore contingent claim prices are not uniquely defined. Our approach is based on Shirakawa's (1991) extension of the HJM model, in which a Wiener noise is generalized into a Wiener-Poisson noise, with a finite number of possible jumps. Shirakawa (1991) assumes the existence of a sufficient number of bonds to hedge away all of the jump risks, and so obtains a unique arbitrage-free pricing measure. Our specification here is slightly more general in that it allows the generalized noise to be maturity-dependent.

Bhar, Chiarella and Tô (2002) demonstrate that attempts to estimate HJM models directly from the instantaneous forward rate specification using the short term futures yield as a proxy would result in non-negligible estimation bias. Therefore, they first derive the evolution of the futures prices by treating the futures contract as a derivative instrument written on the instantaneous forward rate. In this paper we extend the approach of Bhar, Chiarella and Tô (2002) to the HJM model under jump-diffusion and show that if the underlying market variable follows a jump-diffusion process, then the futures prices should also experience jump components. We then use this futures price evolution to estimate our model's parameters using Duan's (1994) likelihood transformation method. Under our specification, the likelihood is well defined, details will be discussed in later sections of the paper.

The theoretical contribution of the paper is, therefore, two-fold. The first is to derive the evolution of futures prices ${ }^{1}$. This evolution can then be used as an input for the Björk and Landén (2002) framework to determine the evolution of options on futures. ${ }^{2}$ The second contribution is to utilize this evolution to obtain a method to estimate the parameters for all HJM models with time-deterministic diffusion and jump volatilities, under the finite jump assumption. This estimation method does not rely on the

\footnotetext{
${ }^{1}$ As far as we are aware, research so far has exclusively focused on bond and option pricing rather than futures contracts.

${ }^{2}$ Björk and Landén (2002) start with a marked point process for the instantaneous forward rate, and assert a marked point process for the futures prices without establishing a link between the two sets of parameters.
} 
Markovianization of the system comprising the spot rate of interest, and equivalently the bond price, which may not always be possible.

We then estimate the jump-diffusion HJM model for four major futures markets around the world, namely the Chicago Mercantile Exchange (CME), the London International Financial and Futures Exchange (LIFFE), the Tokyo International Financial Futures Exchange (TIFFE) and the Sydney Futures Exchange (SFE). The empirical analysis supports the existence of jump components in the CME futures market, as predicted by previous studies of jumps utilizing U.S. data. The SFE futures market also experiences jumps, though the mechanism by which a surprise in information impacts on the Australian market is different from that of the U.S market. On the other hand, we do not find empirical support for the existence of jump components in the U.K market. In the TIFFE market, it seems that the perception of the jump risk is negligible, since the market price of jump risk is found not to be significantly different from zero.

The rest of the paper is organized as follows. Section 2 discusses the class of HJMjump models with which we are working. The evolution of futures prices are derived in Section 3. This evolution is subsequently used to estimate the model's parameters via the likelihood transformation method proposed by Duan (1994), as presented in Section 4. Data and models considered are described in Section 5. We discuss the empirical finding in Section 6. Finally, Section 7 concludes the paper. All technical details are relegated to the Appendices.

\section{MODEL FRAMEWORK}

Consider a financial market characterized by a probability space $(\Omega, \mathcal{F}, \mathcal{P})$. Assume that the probability space carries a standard Wiener process $W$, and $M$ Poisson processes $N_{1}, N_{2}, \ldots, N_{M}$, respectively associated with intensities $v_{1}(t), v_{2}(t), \ldots, v_{M}(t)$. We further assume that the Poisson processes are independent of each other and of the Wiener process.

The dynamic evolution of the instantaneous $T$-maturity forward rate $f(t, T)$ (for $t \leq T \in \mathbb{R}^{+}$) is assumed to be governed by the stochastic differential equation

$$
d f(t, T)=\alpha(t, T) d t+\sigma(t, T) d W(t)+\sum_{m=1}^{M} \delta_{m}(t, T) \gamma_{m} d N_{m}(t)
$$


where $\alpha(t, T)$ and the $\sigma(t, T)$ are respectively the drift and the Wiener diffusion coefficient for the instantaneous forward rate to maturity $T$, whereas $\gamma_{m}(m=1, \ldots, M)$ is the constant jump size that occurs at the $N_{m}$ Poisson jump time. The corresponding jump volatility function is $\delta_{m}(t, T)$.

This model extends the HJM framework by allowing the evolution of the instantaneous forward rate to include discrete jump components in addition to the diffusion process. Each of the noise terms is then scaled by the corresponding volatility functions. The process, is therefore, a special case of the marked point process introduced by Björk, Kabanov and Runggaldier (1997), where the marked space is finite ${ }^{3}$. If we recall one of the very first models that include jump components for forward rate specification, that of Shirakawa (1991) for which the dynamics of the forward rate are given by

$$
d f(t, T)=\alpha^{*}(t, T) d t+\sigma(t, T)\left[d W(t)+\sum_{m=1}^{M} \gamma_{m}\left(d N_{m}(t)-v_{m} d t\right)\right]
$$

we can see that our specification in (2.1) allows for the "generalized noise term" to be maturity dependent. Instead of generalizing the Wiener noise increment $d W(t)$ into a maturity independent Wiener-Poisson noise increment

$$
d W(t)+\sum_{m=1}^{M} \gamma_{m}\left(d N_{m}(t)-v_{m} d t\right)
$$

we generalize the Wiener noise increment into the maturity dependent Wiener-Poisson noise increment

$$
d W(t)+\sum_{m=1}^{M} \frac{\delta_{m}(t, T)}{\sigma(t, T)} \gamma_{m}\left(d N_{m}(t)-v_{m} d t\right)
$$

This generalization has the advantage of giving us greater flexibility to capture empirically the jump components. It has the disadvantage of making the Markovianization of the associated spot rate or bond price difficult, if not impossible ${ }^{4}$. However we do not suffer from this disadvantage since our work is based on the futures prices, and our manipulations to obtain an expression for futures prices do not require Markovianization of the system dynamics.

\footnotetext{
${ }^{3}$ The reason we impose a finite marked space is to ensure market completeness. Details will be discussed later in the section.

${ }^{4}$ For some results on Markovianization of the HJM models under jump-diffusion see Chiarella and Nikitopoulos Sklibosios (2002) and Chiarella and Kwon (2002b).
} 
Under the historical martingale measure $\mathcal{P}$, the instantaneous $T$-maturity forward rate 2.1 evolves according to the stochastic integral equation

$$
\begin{aligned}
f(t, T)= & f(0, T)+\int_{0}^{t} \alpha(u, T) d u+\int_{0}^{t} \sigma(u, T) d W(u) \\
& +\sum_{m=1}^{M} \int_{0}^{t} \delta_{m}(u, T) \gamma_{m} d N_{m}(u),
\end{aligned}
$$

from which the evolution of the instantaneous spot rate can be derived by setting $T=t$, thus

$$
\begin{aligned}
r(t)= & f(0, t)+\int_{0}^{t} \alpha(u, t) d u+\int_{0}^{t} \sigma(u, t) d W(u) \\
& +\sum_{m=1}^{M} \int_{0}^{t} \delta_{m}(u, t) \gamma_{m} d N_{m}(u) .
\end{aligned}
$$

By application of the generalized Itô's lemma (see Appendix A), the dynamics for $P(t, T)$, the price at time $t$ of a bond maturing at time $T$, may be expressed as

$$
\begin{aligned}
d P(t, T)= & P(t, T)\left[r(t)+A(t, T)+\frac{1}{2} S(t, T)^{2}\right] d t+P(t, T) S(t, T) d W(t) \\
& +\sum_{m=1}^{M} P\left(t^{-}, T\right)\left[\mathrm{e}^{D_{m}(t, T)}-1\right] d N_{m}(t)
\end{aligned}
$$

where

$$
\begin{aligned}
& A(t, T) \equiv-\int_{t}^{T} \alpha(t, s) d s \\
& S(t, T) \equiv-\int_{t}^{T} \sigma(t, s) d s
\end{aligned}
$$

and

$$
D_{m}(t, T) \equiv-\int_{t}^{T} \delta_{m}(t, s) \gamma_{m} d s, \text { for } m=1, \ldots, M .
$$

The specification (2.1) limits the jump space to be finite. Björk, Kabanov and Runggaldier (1997) then prove (in theorem 5.6, page 235) that the market is complete (ie. every contingent claim can be replicated by a self-financing portfolio) if the following 2 conditions hold:

(i) For each $t$, the functions $\sigma(t, T)$ and $\delta_{m}(t, T)$ are analytic in the $T$-variable 
(ii) For each $t$, the functions

$$
S(t, T), \quad \mathrm{e}^{D_{m}(t, T)}-1, \quad m=1, \ldots, M,
$$

of the argument $T$ are linearly independent. Furthermore, for each $t$ we can choose the distinct bond maturities arbitrarily, apart from a finite number of values on every compact interval. If all functions above are deterministic and analytic also in the $t$ variable, then the maturities can be chosen to be the same for every $t$.

The completeness of the market implies that the martingale measure is unique (see Corollary 4.8 of Björk, Kabanov and Runggaldier (1997), p229). Under this equivalent martingale measure $\widetilde{\mathcal{P}}$, the bond dynamics are given by

$$
\begin{aligned}
d P(t, T)= & P(t, T) r(t) d t+P(t, T) S(t, T) d \widetilde{W}(t) \\
& +\sum_{m=1}^{M} P\left(t^{-}, T\right)\left[\mathrm{e}^{D_{m}(t, T)}-1\right] d N_{m}(t),
\end{aligned}
$$

where $\widetilde{W}$ is a standard Wiener process and $N_{m}$ is a Poisson process associated with intensity $\lambda_{m}$, for $m=1, \ldots, M$, which can be interpreted as the market price of jump risk, see Shirakawa (1991). The Poisson processes $N_{m}$ remain independent of each other and of the Wiener process $\widetilde{W}$.

Björk, Kabanov and Runggaldier (1997) (Proposition 3.13, p222) show that to eliminate arbitrage opportunities, the relation

$$
\begin{aligned}
\alpha(t, T) & =\sigma(t, T) \int_{t}^{T} \sigma(t, s) d s-\sum_{m=1}^{M} \delta_{m}(t, T) \gamma_{m} \mathrm{e}^{D_{m}(t, T)} \lambda_{m}(t) \\
& \equiv \tilde{\alpha}(t, T)
\end{aligned}
$$

must hold under $\widetilde{\mathcal{P}}$.

Since all derivative instruments can then be priced under the equivalent martingale measure, for model application and pricing purposes, it is sufficient to estimate only the model parameters that appear under the equivalent measure, i.e. the parameters of the volatility coefficients of the Wiener and Poisson processes, and the $\widetilde{\mathcal{P}}$-intensities $\lambda_{m}(t)(m=1, \ldots, M)$. 


\section{EVOLUTION OF FUTURES PRICES UNDER HJM WITH JUMPS}

Let $F\left(t, T_{F}, T_{B}\right)$ be the price at time $t$ of a futures contract maturing at time $T_{F}(>t)$. The contract is written on a pure discount instrument which has a face value of $\$ 1$ and matures at time $T_{B}\left(>T_{F}\right)$. Let the price of this instrument at time $T_{F}$ be $P\left(T_{F}, T_{B}\right)$ and the logarithm value of the price be $V\left(T_{F}, T_{B}\right)$.

Since futures contracts are marked-to-market, it is shown in Cox, Ingersoll and Ross (1981) that the futures price is a martingale under the equivalent measure $\widetilde{\mathcal{P}}$, so that

$$
\begin{aligned}
F\left(t, T_{F}, T_{B}\right) & =\mathbb{E}_{t}^{\widetilde{\mathcal{P}}}\left[F\left(T_{F}, T_{F}, T_{B}\right) \mid \mathcal{F}_{t}\right] \\
& =\mathbb{E}_{t}^{\widetilde{\mathcal{P}}}\left[P\left(T_{F}, T_{B}\right) \mid \mathcal{F}_{t}\right] \\
& =\mathbb{E}_{t}^{\widetilde{\mathcal{P}}}\left[\exp \left(V\left(T_{F}, T_{B}\right)\right) \mid \mathcal{F}_{t}\right] .
\end{aligned}
$$

The $\log$-price $V\left(T_{F}, T_{B}\right)$ under $\widetilde{\mathcal{P}}$ is given by

$$
\begin{aligned}
V\left(T_{F}, T_{B}\right)= & -\int_{T_{F}}^{T_{B}} f\left(T_{F}, s\right) d s \\
= & -\int_{T_{F}}^{T_{B}} f(0, s) d s-\int_{T_{F}}^{T_{B}} \int_{0}^{T_{F}} \tilde{\alpha}(u, s) d u d s \\
& -\int_{T_{F}}^{T_{B}} \int_{0}^{T_{F}} \sigma(u, s) d \widetilde{W}(u) d s-\sum_{m=1}^{M} \int_{T_{F}}^{T_{B}} \int_{0}^{T_{F}} \delta_{m}(u, s) \gamma_{m} d N_{m}(u) d s .
\end{aligned}
$$

By an application of the stochastic Fubini theorem

$$
\begin{aligned}
V\left(T_{F}, T_{B}\right)= & -\int_{T_{F}}^{T_{B}} f(0, s) d s-\int_{0}^{T_{F}} \int_{T_{F}}^{T_{B}} \tilde{\alpha}(u, s) d s d u \\
& -\int_{0}^{T_{F}} \int_{T_{F}}^{T_{B}} \sigma(u, s) d s d \widetilde{W}(u)-\sum_{m=1}^{M} \int_{0}^{T_{F}} \int_{T_{F}}^{T_{B}} \delta_{m}(u, s) \gamma_{m} d s d N_{m}(u) .
\end{aligned}
$$


Therefore ${ }^{5}$

$$
\begin{aligned}
F\left(t, T_{F}, T_{B}\right)= & \mathbb{E}_{t}^{\widetilde{\mathcal{P}}}\left[\exp \left(V\left(T_{F}, T_{B}\right)\right) \mid \mathcal{F}_{t}\right] \\
= & \exp \left[-\int_{T_{F}}^{T_{B}} f(0, s) d s-\int_{0}^{T_{F}} \int_{T_{F}}^{T_{B}} \tilde{\alpha}(u, s) d s d u\right. \\
& \left.\quad-\int_{0}^{t} \int_{T_{F}}^{T_{B}} \sigma(u, s) d s d \widetilde{W}(u)-\sum_{m=1}^{M} \int_{0}^{t} \int_{T_{F}}^{T_{B}} \delta_{m}(u, s) \gamma_{m} d s d N_{m}(u)\right] \\
\times & \mathbb{E}_{t}^{\widetilde{\mathcal{P}}}\left[\exp \left(-\int_{t}^{T_{F}} \int_{T_{F}}^{T_{B}} \sigma(u, s) d s d \widetilde{W}(u)\right)\right] \\
\times & \prod_{m=1}^{M} \mathbb{E}_{t}^{\widetilde{\mathcal{P}}}\left[\exp \left(-\int_{t}^{T_{F}} \int_{T_{F}}^{T_{B}} \delta_{m}(u, s) \gamma_{m} d s d N_{m}(u)\right)\right]
\end{aligned}
$$

due to the independence of the Wiener and the Poisson processes.

The final task is to carry out the expectation operations. The first expectation in (3.1) is a standard result. In fact, Musiela, Turnbull and Wakeman (1992) have used this method to derive the futures price evolution when the market jump components are absent from the market. The second expectation, which involves the increments of

\footnotetext{
${ }^{5}$ The reader is reminded that at time $t$, the integrals from 0 to $t$ of $d \widetilde{W}$ and $d N_{m}(t)$ are realized quantities.
} 
Poisson processes, is evaluated in Appendix B. The final result is

$$
\begin{aligned}
F( & \left.t, T_{F}, T_{B}\right) \\
=\exp & {\left[-\int_{T_{F}}^{T_{B}} f(0, s) d s-\int_{0}^{T_{F}} \int_{T_{F}}^{T_{B}} \tilde{\alpha}(u, s) d s d u\right.} \\
& \left.-\int_{0}^{t} \int_{T_{F}}^{T_{B}} \sigma(u, s) d s d \widetilde{W}(u)-\sum_{m=1}^{M} \int_{0}^{t} \int_{T_{F}}^{T_{B}} \delta_{m}(u, s) \gamma_{m} d s d N_{m}(u)\right] \\
\times & \exp \left[\frac{1}{2} \int_{t}^{T_{F}}\left(\int_{T_{F}}^{T_{B}} \sigma(u, s) d s\right)^{2} d u\right] \\
\times & \prod_{m=1}^{M} \exp \left\{\lambda_{m} \int_{t}^{T_{F}}\left[\exp \left(-\int_{T_{F}}^{T_{B}} \delta_{m}(u, s) \gamma_{m} d s\right)-1\right] d u\right\} \\
=F( & \left., T_{F}, T_{B}\right) \exp \left\{-\frac{1}{2} \int_{0}^{t}\left(\int_{T_{F}}^{T_{B}} \sigma(u, s) d s\right)^{2} d u\right. \\
& -\sum_{m=1}^{M} \lambda_{m}^{t}\left[\exp \left(-\int_{T_{F}}^{T_{B}} \delta_{m}(u, s) \gamma_{m} d s\right)-1\right] d u \\
& \left.-\int_{0}^{t} \int_{T_{F}}^{T_{B}} \sigma(u, s) d s d \widetilde{W}(u)-\sum_{m=1}^{M} \int_{0}^{t} \int_{T_{F}}^{T_{B}} \delta_{m}(u, s) \gamma_{m} d s d N_{m}(u)\right\}
\end{aligned}
$$

From this stochastic integral equation for $F\left(t, T_{F}, T_{B}\right)$, we can derive the corresponding stochastic differential equation

$$
\frac{d F\left(t, T_{F}, T_{B}\right)}{F\left(t^{-}, T_{F}, T_{B}\right)}=\alpha^{(F)}(t, \cdot) d t+\sigma^{(F)}(t, \cdot) d \widetilde{W}(t)+\sum_{m=1}^{M} \delta_{m}^{(F)}(t, \cdot) d N_{m}(t),
$$

where

$$
\begin{aligned}
\sigma^{(F)}(t, \cdot) & \equiv-\int_{T_{F}}^{T_{B}} \sigma(t, s) d s \\
\delta_{m}^{(F)}(t, \cdot) & \equiv \exp \left(-\int_{T_{F}}^{T_{B}} \delta_{m}(t, s) \gamma_{m} d s\right)-1, \\
\alpha^{(F)}(t, \cdot) & \equiv-\sum_{m=1}^{M} \lambda_{m} \delta_{m}^{(F)} .
\end{aligned}
$$

We have treated the futures contract as a derivative instrument written on the forward rates to derive the evolution for the futures price. This evolution is important for two 
practical reasons. First, since futures prices are quoted in the market ${ }^{6}$, the evolution of $F\left(t, T_{F}, T_{B}\right)$ can be used to estimate the parameters of the forward rate specification, which is our main focus here. Second, this evolution may be used as an input for the Björk and Landén (2002) framework to derive the prices of options on futures, such applications however are beyond the scope of the present paper.

\section{The Likelihood Transformation TeChniQue}

The evolution of $F\left(t, T_{F}, T_{B}\right)$ involves the parameters of the forward rate specification. In order to use this evolution to estimate the model parameters, one possible approach is to discretize the stochastic differential equation (3.3). However, the usual Euler discretization may result in inconsistent estimators (see Lo (1988) for detailed discussion). Therefore, we will derive the true likelihood function for futures prices via a state variable, under the likelihood transformation technique of Duan (1994).

The method of Duan only requires one derivative instrument to estimate the underlying model parameters. However, in our framework, for each underlying instrument, there are a number of futures contracts (with different maturities) traded in the market. In order to include all of these futures series in the estimation, we follow the approach of Bhar, Chiarella and Tô (2002) who set up and derive the full information likelihood function for the quoted futures prices in the case of pure Wiener processes. A review of the method will be presented in the first half of this section for completeness, whereas the second half of the section derives the likelihood function needed to handle the case of Wiener-Poisson noise.

\subsection{State variables.}

Assume that for each underlying pure-discount interest rate instrument, there are $K$ futures contracts maturing at times $T_{F k}(k=1,2, \ldots, K)$. The (observable) quoted futures price in the market is $G\left(t, T_{F k}, T_{B k}\right)$, which is linked with $F\left(t, T_{F k}, T_{B k}\right)$ via a function $\eta$

$$
F\left(t, T_{F k}, T_{B k}\right) \equiv \eta\left(G\left(t, T_{F k}, T_{B k}\right)\right)
$$

\footnotetext{
${ }^{6}$ The quoted value in the market is not exactly $F\left(t, T_{F}, T_{B}\right)$ but some value related to $F\left(t, T_{F}, T_{B}\right)$, depending on each exchange convention.
} 
The link between $F$ and $G$ depends on the quoting convention of each exchange. For example, Eurodollar futures prices traded on the Chicago Mercantile Exchange are related to quoted prices according to

$$
\begin{aligned}
F\left(t, T_{F k}, T_{B k}\right) & =\frac{1}{1+\left(1-\frac{G\left(t, T_{F k}, T_{B k}\right)}{100}\right)\left(T_{B k}-T_{F k}\right)} \\
& \equiv \eta\left(G\left(t, T_{F k}, T_{B k}\right)\right) .
\end{aligned}
$$

The quoting convention of other exchanges that are considered in the empirical part of this paper are given in Appendix C. We are considering the case in which all of the futures contracts are written on the same underlying instrument, and therefore the time to maturity of the underlying contract is $T_{B k}-T_{F k}=\tau$ constant for all $k \in[0, K]$.

Let $X\left(t, T_{F k}, T_{B k}\right)$ be a state variable defined by

$$
\begin{aligned}
X\left(t, T_{F k}, T_{B k}\right) & =\ln \left(F\left(t, T_{F k}, T_{B k}\right)\right) \\
& \equiv \zeta\left(F\left(t, T_{F k}, T_{B k}\right)\right) .
\end{aligned}
$$

From (3.2) we have that $X\left(t, T_{F k}, T_{B k}\right)$ satisfies the stochastic integral equation

$$
\begin{aligned}
X\left(t, T_{F k}, T_{B k}\right)= & X\left(0, T_{F k}, T_{B k}\right)+\int_{0}^{t} \mu_{k}(u, \cdot) d u \\
& +\int_{0}^{t} \beta_{k}(u, \cdot) d \widetilde{W}(u)+\sum_{m=1}^{M} \int_{0}^{t} \nu_{m, k}(u, \cdot) d N_{m}(u),
\end{aligned}
$$

where

$$
\begin{aligned}
\mu_{k}(u, \cdot) & \equiv-\frac{1}{2} \beta_{k}^{2}(u, \cdot)-\sum_{m=1}^{M} \lambda_{m}\left[\exp \left(\nu_{m, k}(u, \cdot)\right)-1\right] \\
\beta_{k}(u, \cdot) & \equiv-\int_{T_{F k}}^{T_{B k}} \sigma(u, s) d s \\
\nu_{m, k}(u, \cdot) & \equiv-\int_{T_{F k}}^{T_{B k}} \delta_{m}(u, s) \gamma_{m} d s
\end{aligned}
$$

In order to set up a maximum likelihood estimation procedure, we need to find the density function for the state variable $X$, then transform it successively into the density functions for $F$ and $G$.

\subsection{The likelihood transformation formula.}


Let $X_{j k} \equiv X\left(t_{j}, T_{F k}, T_{B k}\right)$ be an unobservable state variable $k(k=1,2, \ldots, K)$ occurring at time $t_{j}<T_{F k}(j=0,1, \ldots, J)$.

Denote by $\boldsymbol{x}_{j}$ the vector of unobservable state variables occurring at time $t_{j}$, ie. $\boldsymbol{x}_{j}=$ $\left(X_{j 1}, X_{j 2}, \ldots, X_{j K}\right)$. Denote by $\boldsymbol{x}$ the unobservable state vector of size $K(J+1) \times 1$ at time $t_{J}$, ie.

$$
\begin{aligned}
\boldsymbol{x} & =\operatorname{vec}\left(\begin{array}{cccc}
\boldsymbol{x}_{0} & \boldsymbol{x}_{1} & \ldots & \boldsymbol{x}_{J}
\end{array}\right) \\
& =\operatorname{vec}\left(\begin{array}{cccc}
X\left(t_{0}, T_{F 1}, T_{B 1}\right) & X\left(t_{1}, T_{F 1}, T_{B 1}\right) & \ldots & X\left(t_{J}, T_{F 1}, T_{B 1}\right) \\
X\left(t_{0}, T_{F 2}, T_{B 2}\right) & X\left(t_{1}, T_{F 2}, T_{B 2}\right) & \ldots & X\left(t_{J}, T_{F 2}, T_{B 2}\right) \\
\vdots & \vdots & \ddots & \vdots \\
X\left(t_{0}, T_{F K}, T_{B K}\right) & X\left(t_{1}, T_{F K}, T_{B K}\right) & \ldots & X\left(t_{J}, T_{F K}, T_{B K}\right)
\end{array}\right),
\end{aligned}
$$

where vec is the standard matrix operator that, when applied to a matrix, transforms the matrix into a vector by stacking the columns of the matrix on top of each other.

Denote the density function of $\boldsymbol{X}$ by

$$
p_{\boldsymbol{X}}(\boldsymbol{x} ; \boldsymbol{\theta})=p_{\boldsymbol{X}}\left(\boldsymbol{x}_{0}, \boldsymbol{x}_{1}, \ldots, \boldsymbol{x}_{J} ; \boldsymbol{\theta}\right),
$$

where $\theta \in \Theta$ is the parameter vector of interest.

Suppose that a transformation $\boldsymbol{\Xi}$ exists, which applied to $\boldsymbol{X}$, produces a vector $\boldsymbol{Z}$ that is observable in the market, so that

$$
\boldsymbol{Z}=\boldsymbol{\Xi}(\boldsymbol{X} ; \boldsymbol{\theta}): \mathbb{R}^{K(J+1) \times 1} \rightarrow \mathbb{R}^{K(J+1) \times 1},
$$

where

$$
\begin{aligned}
\boldsymbol{z} & =\operatorname{vec}\left(\begin{array}{cccc}
\boldsymbol{z}_{0} & \boldsymbol{z}_{1} & \ldots & \boldsymbol{z}_{J}
\end{array}\right) \\
& =\operatorname{vec}\left(\begin{array}{cccc}
Z_{01} & Z_{11} & \ldots & Z_{J 1} \\
Z_{02} & Z_{12} & \ldots & Z_{J 2} \\
\vdots & \vdots & \ddots & \vdots \\
Z_{0 K} & Z_{1 K} & \ldots & Z_{J K}
\end{array}\right) .
\end{aligned}
$$

Assume that this transformation is one-to-one for every $\boldsymbol{\theta} \in \Theta$.

Since $\boldsymbol{\Xi}$ is one-to-one, there exists an inverse $\Xi^{-1}=\Psi(Z ; \theta)$. Applying the standard change of variable technique we obtain the density function for $Z$ as

$$
p_{\boldsymbol{Z}}(\boldsymbol{z}, \boldsymbol{\theta})=p_{\boldsymbol{X}}(\boldsymbol{\Psi}(\boldsymbol{z} ; \boldsymbol{\theta})) \times|\mathrm{J}(\boldsymbol{\Psi}(\boldsymbol{z} ; \boldsymbol{\theta}))|,
$$


where $\mathrm{J}$ is the Jacobian of the transformation from $\boldsymbol{X}$ to $\boldsymbol{Z}$, ie.

$$
\mathrm{J}(\boldsymbol{\Psi}(\boldsymbol{z} ; \boldsymbol{\theta}))=\left|\frac{\partial \boldsymbol{\Psi}(\boldsymbol{z} ; \boldsymbol{\theta})}{\partial \boldsymbol{z}^{\prime}}\right| .
$$

Duan (1994) proves that if the transformation is on an element-by-element basis, ie. $Z_{j k}=\Xi_{j k}\left(X_{j k}\right)$ (and $X_{j k}=\Psi_{j k}\left(Z_{j k}\right)$ ) for all $j \in[0, J]$ and $k \in[1, K]^{7}$, then the first-derivative matrix is diagonal, therefore

$$
\mathrm{J}(\boldsymbol{\Psi}(\boldsymbol{z} ; \boldsymbol{\theta}))=\prod_{j=0}^{J} \prod_{k=1}^{K} \frac{d \Psi_{j k}\left(Z_{j k} ; \boldsymbol{\theta}\right)}{d Z_{j k}} .
$$

Furthermore, if $\boldsymbol{X}$ is “joint-Markovian", ie.

$$
p_{\boldsymbol{X}}\left(\boldsymbol{x}_{0}, \boldsymbol{x}_{1}, \ldots, \boldsymbol{x}_{J} ; \boldsymbol{\theta}\right)=p_{\boldsymbol{X}}\left(\boldsymbol{x}_{0}, t_{0} ; \boldsymbol{\theta}\right) \prod_{j=1}^{J} p_{\boldsymbol{X}}\left(\boldsymbol{x}_{j}, t_{j} \mid \boldsymbol{x}_{j-1}, t_{j-1} ; \boldsymbol{\theta}\right)
$$

then upon substitution, the likelihood for $Z$ can be compactly written as

$$
p_{\boldsymbol{Z}}\left(\boldsymbol{z}_{0}, \boldsymbol{z}_{1}, \ldots, \boldsymbol{z}_{J} ; \boldsymbol{\theta}\right)=p_{\boldsymbol{Z}}\left(\boldsymbol{z}_{0}, t_{0} ; \boldsymbol{\theta}\right) \prod_{j=1}^{J} p_{\boldsymbol{Z}}\left(\boldsymbol{z}_{j}, t_{j} \mid \boldsymbol{z}_{j-1}, t_{j-1} ; \boldsymbol{\theta}\right),
$$

where

$$
\begin{aligned}
p_{\boldsymbol{Z}}\left(\boldsymbol{z}_{0}, t_{0} ; \boldsymbol{\theta}\right) & =p_{\boldsymbol{X}}\left(\boldsymbol{\Psi}_{0}\left(\boldsymbol{y}_{\mathbf{0}}\right), t_{0} ; \boldsymbol{\theta}\right) \times\left|\mathrm{J}\left(\boldsymbol{\Psi}_{0}\left(\boldsymbol{z}_{0} ; \boldsymbol{\theta}\right)\right)\right|, \\
p_{\boldsymbol{Z}}\left(\boldsymbol{z}_{j}, t_{j} \mid \boldsymbol{z}_{j-1}, t_{j-1} ; \boldsymbol{\theta}\right) & =p_{\boldsymbol{X}}\left(\boldsymbol{\Psi}_{j}\left(\boldsymbol{z}_{j}\right), t_{j} \mid \boldsymbol{\Psi}_{j-1}\left(\boldsymbol{z}_{j-1}\right), t_{j-1} ; \boldsymbol{\theta}\right) \times\left|\mathrm{J}\left(\boldsymbol{\Psi}_{j}\left(\boldsymbol{z}_{j} ; \boldsymbol{\theta}\right)\right)\right|,
\end{aligned}
$$

and

$$
\mathrm{J}\left(\boldsymbol{\Psi}\left(\boldsymbol{z}_{j} ; \boldsymbol{\theta}\right)\right)=\prod_{k=1}^{K} \frac{d \Psi_{j k}\left(Z_{j k} ; \boldsymbol{\theta}\right)}{d Z_{j k}} \quad(j=0,1, \ldots, J) .
$$

\subsection{Full information likelihood for quoted futures prices.}

Before deriving the likelihood function, we introduce into the system a measurement error, ie. a deviation of market quoted value and the true model value. We will assume that on average, this measurement error brings zero return to the trading of futures contracts. Therefore, a new Wiener process $\widetilde{\varepsilon}_{k}$, which is independent of the process driving the uncertainty of the forward rate, is introduced into the evolution of

\footnotetext{
${ }^{7}$ This assumption is not restrictive in the financial market context, since each financial instrument is usually determined by only one particular underlying variable. For example, a bond price at a time is determined by the interest rate of a particular maturity at that particular time.
} 
$F\left(t, T_{F k}, T_{B k}\right)$, so (3.3) becomes

$$
\begin{aligned}
\frac{d F\left(t, T_{F}, T_{B}\right)}{F\left(t-, T_{F}, T_{B}\right)}= & \alpha^{(F)}(t, \cdot) d t+\sigma^{(F)}(t, \cdot) d \widetilde{W}(t) \\
& +\sigma_{\varepsilon} d \widetilde{\varepsilon}_{k}(t)+\sum_{m=1}^{M} \delta_{m}^{(F)}(t, \cdot) d N_{m}(t),
\end{aligned}
$$

We assume that there is no correlation between the return-measurement error on contracts with different maturities (ie. no correlation between $d \widetilde{\varepsilon}_{k}$ for $k=1, \ldots, K$ ). Since there is no reason to believe the error in one particular contract is larger than in others, we use the same error-volatility value $\sigma_{\varepsilon}$ for all contracts ${ }^{8}$.

The evolution of our state variable $X$, incorporating this measurement error, is

$$
\begin{aligned}
X\left(t, T_{F k}, T_{B k}\right) & =X\left(0, T_{F k}, T_{B k}\right)+\int_{0}^{t}\left(\mu_{k}(u, \cdot)-\frac{1}{2} \sigma_{\varepsilon}^{2}\right) d u \\
+ & \int_{0}^{t} \beta_{k}(u, \cdot) d \widetilde{W}(u)+\int_{0}^{t} \sigma_{\varepsilon} d \widetilde{\varepsilon}_{k}(u)+\sum_{m=1}^{M} \int_{0}^{t} \nu_{m, k}(u, \cdot) d N_{m}(u) .
\end{aligned}
$$

Suppose that the process is sampled at $J+1$ discrete points in time $t_{0}, t_{1}, \ldots, t_{n}$ (not necessarily equally spaced apart). Due to the Markovian nature of the system (ie. the stochastic processes for $X\left(t, T_{F k}, T_{B k}\right)$ for $\left.k=1, \ldots, K\right)$, the likelihood function for joint observation $\boldsymbol{x}^{9}$, for a given parameter vector of interest $\boldsymbol{\theta} \in \boldsymbol{\Theta}$, is

$$
p_{\boldsymbol{X}}\left(\boldsymbol{x}_{0}, \boldsymbol{x}_{1}, \ldots, \boldsymbol{x}_{J} ; \boldsymbol{\theta}\right)=p_{\boldsymbol{X}}\left(\boldsymbol{x}_{0}, t_{0} ; \boldsymbol{\theta}\right) \prod_{j=1}^{J} p_{\boldsymbol{X}}\left(\boldsymbol{x}_{j}, t_{j} \mid \boldsymbol{x}_{j-1}, t_{j-1} ; \boldsymbol{\theta}\right) .
$$

The specification (4.4) implies that the transitional likelihood function for $X$ will have a form of a (Poisson) mixture of normal distributions. Our assumption that allows for only finite (in fact only one) jump size at each jump time, rather than jumps being

\footnotetext{
${ }_{8}^{8}$ In practice, this error volatility should be small in order and magnitude compared to the diffusion volatility, so that any attempt to set up an arbitrage portfolio to trade on this uncertainty source will not result in profits after transaction costs are taken into account.
}

${ }^{9}$ Recall that

$$
\boldsymbol{x}=\operatorname{vec}\left(\begin{array}{llll}
\boldsymbol{x}_{0} & \boldsymbol{x}_{1} & \ldots & \boldsymbol{x}_{J}
\end{array}\right)=\operatorname{vec}\left(\begin{array}{cccc}
X_{01} & X_{11} & \ldots & X_{J 1} \\
X_{02} & X_{12} & \ldots & X_{J 2} \\
\vdots & \vdots & \ddots & \vdots \\
X_{0 K} & X_{1 K} & \ldots & X_{J K}
\end{array}\right) \text {, }
$$

and $X_{j k} \equiv X\left(t_{j}, T_{F k}, T_{B k}\right)$, for $j=0,1, \ldots, J$ and $k=1,2, \ldots, K$ 
drawn from a (normal) distribution, ensures that the transitional likelihood function is bounded, and therefore, well defined ${ }^{10}$.

The transitional likelihood function is

$$
\begin{aligned}
& p_{\boldsymbol{X}}\left(\boldsymbol{x}_{j}, t_{j} \mid \boldsymbol{x}_{j-1}, t_{j-1} ; \boldsymbol{\theta}\right) \\
= & \sum_{n_{1}=0}^{\infty} \sum_{n_{2}=0}^{\infty} \ldots \sum_{n_{M}=0}^{\infty}\left(\mathrm{e}^{-\lambda_{1} \Delta t} \frac{\left(\lambda_{1} \Delta t\right)^{n_{1}}}{n_{1} !}\right) \ldots\left(\mathrm{e}^{-\lambda_{M} \Delta t} \frac{\left(\lambda_{M} \Delta t\right)^{n_{M}}}{n_{M} !}\right) \Phi\left(\boldsymbol{x}_{j} ; \boldsymbol{a}_{j}, \boldsymbol{\Omega}_{j}\right),
\end{aligned}
$$

where $\Delta t=t_{j}-t_{j-1}$ and $\Phi$ is a multivariate Gaussian density defined by

$$
\begin{aligned}
\Phi\left(\boldsymbol{x}_{j} ; \boldsymbol{a}_{j}, \boldsymbol{\Omega}_{j}\right)= & (2 \pi)^{-\frac{K}{2}}\left|\boldsymbol{\Omega}_{j}\right|^{-\frac{1}{2}} \\
& \exp \left(-\frac{1}{2}\left(\boldsymbol{x}_{\boldsymbol{j}}-\boldsymbol{x}_{j-1}-\boldsymbol{a}_{j}\right)^{\prime} \boldsymbol{\Omega}_{j}^{-1}\left(\boldsymbol{x}_{\boldsymbol{j}}-\boldsymbol{x}_{j-1}-\boldsymbol{a}_{j}\right)\right),
\end{aligned}
$$

whose mean and variance are

$$
\begin{aligned}
& \boldsymbol{a}_{j}=\left(\begin{array}{llll}
a_{j 1} & a_{j 2} & \ldots & a_{j K}
\end{array}\right)^{\prime}, \\
& \boldsymbol{\Omega}_{j}=\left(\begin{array}{cccc}
b_{j(11)} & b_{j(12)} & \ldots & b_{j(1 K)} \\
b_{j(21)} & b_{j(22)} & \ldots & b_{j(2 K)} \\
\vdots & \vdots & \ddots & \vdots \\
b_{j(K 1)} & b_{j(K 2)} & \ldots & b_{j(K K)}
\end{array}\right) \text {, }
\end{aligned}
$$

and the matrix elements are defined by

$$
\begin{aligned}
a_{j k} & =\int_{t_{j-1}}^{t_{j}}\left(\mu_{k}(u)-\frac{1}{2} \sigma_{\varepsilon}^{2}+\sum_{m=1}^{M} n_{m} \nu_{m k}(u)\right) d u, \\
b_{j(k k)} & =\int_{t_{j-1}}^{t_{j}}\left(\beta_{k}^{2}(u)+\sigma_{\varepsilon}^{2}\right) d u, \\
b_{j\left(k_{1} k_{2}\right)} & =\int_{t_{j-1}}^{t_{j}} \beta_{k_{1}}(u) \beta_{k_{2}}(u) d u \text { for } k_{1} \neq k_{2} .
\end{aligned}
$$

In this paper, rather than using the exact Poisson mixture of Gaussian densities, we use the approximate Bernoulli mixture of Gaussian densities so as to obtain a practically and economically implementable model. The approximation is the result of

\footnotetext{
${ }^{10}$ For a discussion of the unboundedness of the mixture of normal distributions, the reader is referred to Kiefer (1978), Quandt and Ramsey (1978), Honoré (1998), chapter 22 of Hamilton (1994) and the references therein.
} 
ignoring all of the values of order higher than $(d t)^{2}$ in the transitional likelihood function. If the time interval is short (say 1 day) and the Poisson intensity is small, the two densities are practically indistinguishable. The transitional likelihood function for $X$ under this Bernoulli approximation becomes

$$
\begin{aligned}
& p_{\boldsymbol{X}}\left(\boldsymbol{x}_{j}, t_{j} \mid \boldsymbol{x}_{j-1}, t_{j-1} ; \boldsymbol{\theta}\right) \\
= & \sum_{m=1}^{M} \lambda_{m} \Delta t \Phi\left(\boldsymbol{x}_{j} ; \boldsymbol{a}_{j(m)}, \boldsymbol{\Omega}_{j}\right)+\left(1-\sum_{m=1}^{M} \lambda_{m} \Delta t\right) \Phi\left(\boldsymbol{x}_{j} ; \boldsymbol{a}_{j(0)}, \boldsymbol{\Omega}_{j}\right),
\end{aligned}
$$

where

$$
\boldsymbol{a}_{j(m)}=\left(\begin{array}{llll}
a_{j 1(m)} & \left.a_{j 2(m)} \quad \ldots \quad a_{j K(m)}\right)^{\prime}, \quad \text { for } m=0,1, \ldots, M,
\end{array}\right.
$$

and the matrix elements are defined by

$$
\begin{aligned}
a_{j k(0)} & =\int_{t_{j-1}}^{t_{j}}\left(\mu_{k}(u)-\frac{1}{2} \sigma_{\varepsilon}^{2}\right) d u \\
a_{j k(m)} & =\int_{t_{j-1}}^{t_{j}}\left(\mu_{k}(u)-\frac{1}{2} \sigma_{\varepsilon}^{2}+\nu_{m k}(u)\right) d u \quad \text { for } m \neq 0 .
\end{aligned}
$$

The $\log$ likelihood function for the state variable $\boldsymbol{X}$, based on the observation $\boldsymbol{x}$ is

$$
\mathcal{L}_{\boldsymbol{X}}(\boldsymbol{\theta})=\sum_{j=1}^{J} \ln \left(p_{\boldsymbol{X}}\left(\boldsymbol{x}_{j}, t_{j} \mid \boldsymbol{x}_{j-1}, t_{j-1} ; \boldsymbol{\theta}\right)\right)
$$

Recall that there exists a transformation from $\boldsymbol{X}$ to $\boldsymbol{F}$ (see (4.3)) with inverse function $\zeta$. It is clear that this transformation is on element-by-element basis. Applying the transformation formula, the likelihood function for $\boldsymbol{F}$ is

$$
\mathcal{L}_{\boldsymbol{F}}(\boldsymbol{\theta})=\sum_{j=1}^{J} \ln \left(p_{\boldsymbol{X}}\left(\boldsymbol{\zeta}\left(\boldsymbol{F}_{j}\right), t_{j} \mid \boldsymbol{\zeta}\left(\boldsymbol{F}_{j-1}\right), t_{j-1} ; \boldsymbol{\theta}\right)\right)+\sum_{j=1}^{J} \ln \left|\frac{\partial \boldsymbol{\zeta}_{j}\left(\boldsymbol{F}_{j} ; \boldsymbol{\theta}\right)}{\partial \boldsymbol{F}_{j}}\right| .
$$

Applying for a second time the transformation from $\boldsymbol{F}$ to $\boldsymbol{G}$, the quoted futures price in the market, with the inverse transformation function $\boldsymbol{\eta}$ (see (4.1)) results in the log likelihood function

$$
\mathcal{L}_{\boldsymbol{G}}(\boldsymbol{\theta})=\sum_{j=1}^{J} \ln \left(p_{\boldsymbol{F}}\left(\boldsymbol{\eta}\left(\boldsymbol{G}_{j}\right), t_{j} \mid \boldsymbol{\eta}\left(\boldsymbol{G}_{j-1}\right), t_{j-1} ; \boldsymbol{\theta}\right)\right)+\sum_{j=1}^{J} \ln \left|\frac{\partial \boldsymbol{\eta}_{j}\left(\boldsymbol{G}_{j} ; \boldsymbol{\theta}\right)}{\partial \boldsymbol{G}_{j}}\right| .
$$

Finally, it should be noted that futures prices at different maturities are less than perfectly correlated with each other under a stochastic setting. Therefore, the full 
information maximum likelihood method afore-described, which is applied to pooled time series and cross-sectional data, will allow us to exploit the full information content along the yield curve.

\section{Models AND DATA}

5.1. Models. The HJM model given by (2.1) is determined once the volatility functions associated with the diffusion and jump processes are specified.

For the diffusion component, we choose the "hump-volatility" curve specification

$$
\sigma(t, T)=\left[\sigma_{0}+\sigma_{1}(T-t)\right] \exp (-\kappa(T-t))
$$

which nests the exponential model (Hull and White (1990) extended Vasicek model), the linear absolute model considered in Amin and Morton (1994) and the absolute (constant) model of Ho and Lee (1986).

For the jump component, we distinguish between two types of information surprise. The first one is of the macroeconomic type that will affect the whole yield curve more or less equally. The second one is less prominent, and will affect some sections of the yield curve more than the others. Therefore, we choose an exponential form for one jump volatility and a unit volatility for the other jumps to scale up the two assumed constant jump intensities under the risk-neutral measure. Mathematically more precisely, we have set $M=2, \lambda_{1}$ and $\lambda_{2}$ constant, $\delta_{1}(t, T)=\exp \left(-\kappa_{1}(T-t)\right)$, and $\delta_{2}(t, T)=1$.

Under these specifications, both the diffusion volatility and the jump volatility are functions of time-to-maturity $T-t$, but not functions of a specific calender time $t$. Due to the independence of $T \mathrm{e}^{-\kappa T}$ and the $\mathrm{e}^{-\kappa_{m} T}$, the condition (2.4) for market completeness and uniqueness of the equivalent martingale measure holds. Furthermore, at any time $t$, the investment opportunities in the market are spanned by $M+1$ basic bonds with different maturities.

To find the analytical likelihood function for the quoted futures prices, we need to perform the integrations in (4.8), (4.9), (4.5), and (4.6), ie. find each of the elements in the drift and covariance matrix, and substitute them into the final likelihood function (4.7), (4.10), (4.11) and (4.12). Details of these calculations can be found in Appendix D. 
5.2. Data. We carry out our empirical investigation using 3-month futures contracts traded on 4 major exchanges from different parts of the world. We choose the Eurodollar futures contract traded on the Chicago Mercantile Exchange (CME), the Sterling futures contract traded on the London International Financial and Futures Exchange (LIFFE), the 90-day Bank Accepted Bills futures contract traded on the Sydney Futures Exchange (SFE) and the Euroyen futures contract traded on the Tokyo International Financial Futures Exchange (TIFFE).

All of the data have been obtained from the Datastream ${ }^{T M}$ database. The sample period spans 14 years from January 1988 to December 2001, except for the Euroyen futures contract traded on TIFFE, the available data is from September 1989. We choose our sample period to start from 1988 since including 1987 in the sample might bias the result in favour of finding jumps.

Since a futures contract has a relatively short life, we roll over futures contracts along the 14-year sample period. Take the Eurodollar futures contract on the CME as an example. Sample observations for the period January 2000 to December 2000 consist of 6 different contracts: March 2001, December 2001, September 2002, June 2003, March 2004 and December 2004; whereas the sample observations for the period January 2001 to December 2001 consist of a different set of 6 contracts: March 2002, December 2002, September 2003, June 2004, March 2005 and December 2005 (see Figure 1). It should be noted that the sets of contracts used are spaced 3 quarters apart to ensure sufficient variation in the futures prices, and the contracts are selected until there is no longer sufficient liquidity.

Since each exchange has a different liquidity level, the number of contracts used at each point in time will be different, and the time span of each set of contracts to be rolled over will also be different. The Eurodollar contract traded on the CME is the most liquid one, and therefore we use on average 5 different contracts in our estimation at each point of time. In the SFE and LIFFE case, we use on average 2 contracts, and only 1 contract is used in the case of the TIFFE.

\section{EMPIRICAL RESUlts}

All of our empirical work was carried out using $\mathrm{Ox}^{T M}$, a matrix-oriented programming language ${ }^{11}$.

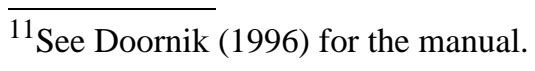


FIGURE 1. Research design - Futures contracts used in sample period January 2001 - December 2001.

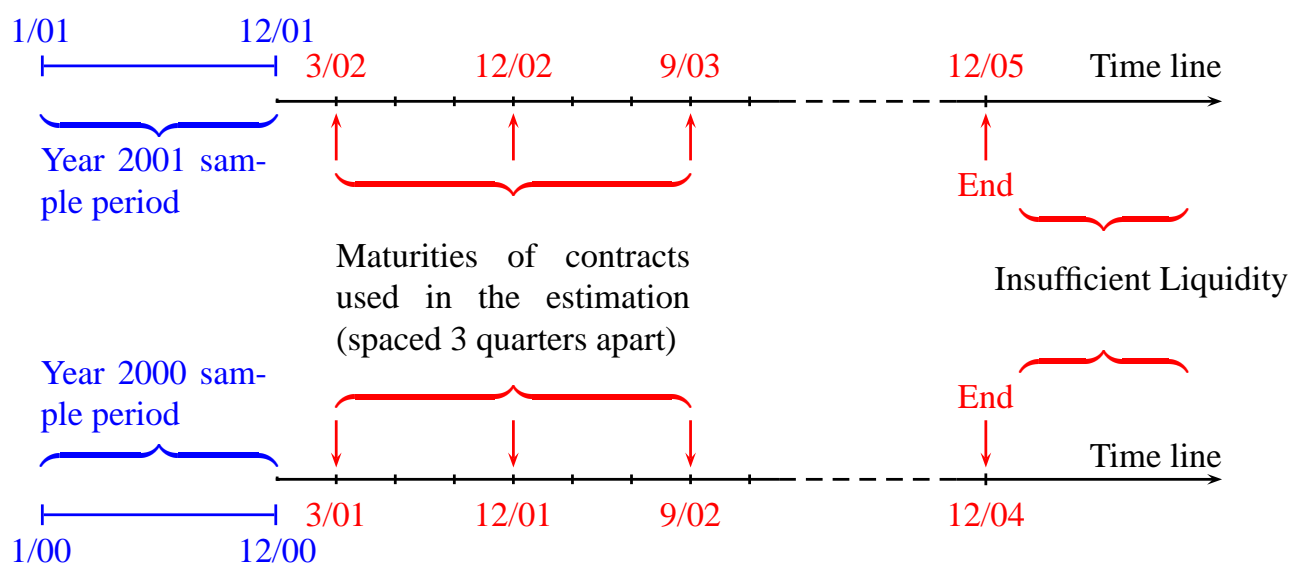

6.1. The estimated models. We ran the estimation for the 3-month futures contracts traded on the 4 exchanges. In the case of the TIFFE, we broke the full sample period into two subsamples, one from 1989-1994, the other from 1996-2001. In 1995, in the Japanese market, there was a sharp fall of the interest rate from $2.5 \%$ to $0.5 \%$. From 1996 to 2001, the level of interest rate remained very low, at an average level of $0.4 \%$.

The empirical results suggested that we did not need two jump processes to capture the effect of surprises on the market. When two jump processes were present together, the estimated values of the parameters associated with the jump components became insignificant. Therefore, we re-estimated our model with only one jump process. The volatility associated with this jump takes the form of an exponential function, which nests the unity case. Depending on the value of the decay factor of the exponential function (ie. significantly different from zero or not), we can distinguish between the two mechanisms of spreading the effect of surprise shocks.

The model estimated parameters are given in Table 1 . The symbol * next to the estimated parameters indicates a significant value at $95 \%$ level of confidence. Table 2 presents the estimation results for more parsimonious models where all of the insignificant parameters have been taken out ${ }^{12}$.

\footnotetext{
$\overline{{ }^{12} \text { We note that }}$ our computer program seems to have difficulty finding the global maxima for $\lambda_{1}$. Even though the significance of the estimate does not change, the point estimate tends to converge to a local maxima, depending on the starting values.
} 
TABle 1. Estimated Parameters for 1 Wiener - 1 Poisson Model

This table reports the estimated parameter values for the model with humped diffusion volatility and exponential jump volatility. The corresponding robust asymptotic standard errors are given in square parenthesis under the estimated values. The * next to the estimated value indicates a significance level lower than $5 \%$.

\begin{tabular}{c|ccccc}
\hline \multirow{3}{*}{ Parameter } & $\begin{array}{c}\text { CME } \\
1988-2001\end{array}$ & $\begin{array}{c}\text { SFE } \\
1988-2001\end{array}$ & $\begin{array}{c}\text { LIFFE } \\
1988-2001\end{array}$ & $\begin{array}{c}\text { TIFFE } \\
1989-1994\end{array}$ & $\begin{array}{c}\text { TIFFE } \\
1996-2001\end{array}$ \\
\hline \multirow{3}{*}{$\hat{\sigma}_{0}$} & $0.0093^{*}$ & $0.0107^{*}$ & $0.0141^{*}$ & $0.0205^{*}$ & $0.0449^{*}$ \\
& {$[0.0005]$} & {$[0.0012]$} & {$[0.0013]$} & {$[0.0052]$} & {$[0.0191]$} \\
$\hat{\sigma}_{1}$ & $0.0040^{*}$ & $0.0087^{*}$ & $1.1 \times 10^{-8}$ & $-0.0896^{*}$ & $-0.1026^{*}$ \\
& {$[0.0006]$} & {$[0.0034]$} & {$\left[1.6 \times 10^{-5}\right]$} & {$[0.0219]$} & {$[0.0456]$} \\
$\hat{\kappa}$ & $0.2403^{*}$ & $0.3101^{*}$ & 0.0662 & $2.7053^{*}$ & $2.6569^{*}$ \\
& {$[0.0198]$} & {$[0.1038]$} & {$[0.0509]$} & {$[0.3559]$} & {$[0.6252]$} \\
$\hat{\gamma}_{1}$ & $0.0057^{*}$ & $0.0056^{*}$ & 0.0001 & $0.0013^{*}$ & $6.5 \times 10^{-5 *}$ \\
& {$[0.0009]$} & {$[0.0006]$} & {$[0.0013]$} & {$[0.0005]$} & {$\left[7.5 \times 10^{-6}\right]$} \\
$\hat{\kappa}_{1}$ & $0.2343^{*}$ & 0.0755 & 0.1991 & 0.0978 & -5.7900 \\
& {$[0.1415]$} & {$[0.1470]$} & {$[0.9190]$} & {$[0.7183]$} & {$[2.0179]$} \\
$\hat{\lambda}_{1}$ & $1.9993^{*}$ & $2.0001^{*}$ & 1.9991 & 1.9714 & 1.9718 \\
& {$[0.6502]$} & {$[0.7334]$} & {$[16.331]$} & {$[3.1780]$} & {$[2.9737]$} \\
$\hat{\sigma}_{\epsilon}$ & $0.0008^{*}$ & $0.0009^{*}$ & $0.0010^{*}$ & $0.0015^{*}$ & $0.0008^{*}$ \\
& {$\left[1.7 \times 10^{-5}\right]$} & {$\left[2.4 \times 10^{-5}\right]$} & {$\left[6.4 \times 10^{-5}\right]$} & {$[0.0001]$} & {$\left[5.8 \times 10^{-5}\right]$} \\
\hline
\end{tabular}

In the CME, SFE and TIFFE markets, the diffusion volatility was found to be humped-shaped, whereas in the LIFFE we did not find supporting evidence for humped or exponential shapes. We re-estimated the model for the LIFFE with a constant volatility specification (ie. with the restriction that $\sigma_{1}=0$ and $\kappa=0$, and with the presence of the jump component), the resulting estimated values of parameters associated with jump component remained insignificant, and there was no significant loss of likelihood value. 
TABLE 2. Estimated Parameters for Restricted Model

This table reports the estimated parameters value for the restricted jumpdiffusion model. The robust asymptotic standard errors are given in square parenthesis. The * next to the estimated parameter value indicates a significance level lower than 5\%. The likelihood ratio statistics are also reported.

\begin{tabular}{|c|c|c|c|c|c|}
\hline & $\begin{array}{c}\text { CME } \\
1988-2001 \\
\end{array}$ & $\begin{array}{c}\text { SFE } \\
1988-2001 \\
\end{array}$ & $\begin{array}{c}\text { LIFFE } \\
1988-2001 \\
\end{array}$ & $\begin{array}{c}\text { TIFFE } \\
1989-1994 \\
\end{array}$ & $\begin{array}{c}\text { TIFFE } \\
1996-2001 \\
\end{array}$ \\
\hline$\hat{\sigma}_{0}$ & $\begin{array}{l}0.0093^{*} \\
{[0.0005]}\end{array}$ & $\begin{array}{c}0.0107 * \\
{[0.0012]}\end{array}$ & $\begin{array}{l}0.0132 * \\
{[0.0006]}\end{array}$ & $\begin{array}{c}0.0200^{*} \\
{[0.0051]}\end{array}$ & $\begin{array}{l}0.0436 * \\
{[0.0187]}\end{array}$ \\
\hline$\hat{\sigma}_{1}$ & $\begin{array}{l}0.0040 * \\
{[0.0006]}\end{array}$ & $\begin{array}{l}0.0087 * \\
{[0.0034]}\end{array}$ & $\begin{array}{l}- \\
-\end{array}$ & $\begin{array}{l}-0.0883 * \\
{[0.0216]}\end{array}$ & $\begin{array}{l}-0.1002 * \\
{[0.0447]}\end{array}$ \\
\hline$\hat{\kappa}$ & $\begin{array}{l}0.2403^{*} \\
{[0.0198]}\end{array}$ & $\begin{array}{l}0.3098^{*} \\
{[0.1039]}\end{array}$ & $\begin{array}{l}- \\
-\end{array}$ & $\begin{array}{l}2.6962 * \\
{[0.3549]}\end{array}$ & $\begin{array}{l}2.6146 * \\
{[0.6256]}\end{array}$ \\
\hline$\hat{\gamma}_{1}$ & $\begin{array}{l}0.0057 * \\
{[0.0009]}\end{array}$ & $\begin{array}{c}0.0053^{*} \\
{[0.0001]}\end{array}$ & $\begin{array}{l}- \\
-\end{array}$ & $\begin{array}{l}- \\
-\end{array}$ & $\begin{array}{l}- \\
-\end{array}$ \\
\hline$\hat{\kappa}_{1}$ & $\begin{array}{l}0.2343 * \\
{[0.1415]}\end{array}$ & $\begin{array}{l}- \\
-\end{array}$ & - & $\begin{array}{l}- \\
-\end{array}$ & $\begin{array}{l}- \\
-\end{array}$ \\
\hline$\hat{\lambda}_{1}$ & $\begin{array}{l}1.9993 * \\
{[0.6502]}\end{array}$ & $\begin{array}{c}1.9998 * \\
{[0.7051]}\end{array}$ & - & $\begin{array}{l}- \\
-\end{array}$ & $\begin{array}{l}- \\
-\end{array}$ \\
\hline$\hat{\sigma}_{\epsilon}$ & $\begin{array}{c}0.0008 * \\
{\left[1.7 \times 10^{-5}\right]}\end{array}$ & $\begin{array}{c}0.0009 * \\
{\left[2.4 \times 10^{-5}\right]}\end{array}$ & $\begin{array}{c}0.0010^{*} \\
{\left[6.6 \times 10^{-5}\right]}\end{array}$ & $\begin{array}{l}0.0015 * \\
{[0.0001]}\end{array}$ & $\begin{array}{c}0.0008^{*} \\
{\left[5.7 \times 10^{-5}\right]}\end{array}$ \\
\hline $\begin{array}{l}\text { Likelihood } \\
\text { Ratio Stat. }\end{array}$ & - & 0.6 & - & - & - \\
\hline
\end{tabular}

The three parameters $\gamma_{1}, \kappa_{1}$ and $\lambda_{1}$ identify the jump component. The magnitude of the jump volatility is shown via $\gamma_{1}$, whereas $\kappa_{1}$ indicates the mechanism by which the effect of jump components are spread across the maturities. The parameter $\lambda_{1}$ can be interpreted as the market price of jump risk, and it should be stressed that $\lambda_{1}$ is not the true jump intensity under the historical measure. However, it is this market 
price of jump risk, not the true intensity, that matters for pricing derivatives (within the framework of a discrete number of jumps assumed in this paper).

In the CME and SFE markets, both $\hat{\lambda}_{1}$ and $\hat{\gamma}_{1}$ are significantly different from zero, supporting the presence of a jump noise component and a market price of jump risk. However, the mechanism by which a surprise in information impacts on the Australian market is different from that of the U.S. market. The Australian market is a small market which is affected substantially by outside factors, in particular what is happening in the U.S. market. The insignificant value of $\hat{\kappa}_{1}$, and the likelihood ratio test for the restriction of $\kappa_{1}=0$ in Table 2, suggest that a surprise in information will affect the whole of the Australian yield curve more or less equally. On the other hand, in the U.S market, a surprise in information has a large impact on the volatility of the short-term section of the yield curve, and this impact dies out gradually along the yield curve towards the longer maturity section. The rate of decrease is reflected in the decay factor $\hat{\kappa}_{1}$. Borrowing a term from the time series analysis literature, as Raj, Sim and Thurston (1997) have done, we define the half life of the shock as the difference in the time to maturity of two forward rates, so that one undergoes double the effect of the other from the same shock, ie.

$$
\gamma_{1} \exp ^{-\kappa_{1}\left(T_{1}-t\right)}=2 \gamma_{1} \exp ^{-\kappa_{1}\left(T_{2}-t\right)}
$$

from which

$$
T_{2}-T_{1}=\frac{\ln 2}{\kappa_{1}}=2.96 \text { years. }
$$

As calculated above, the two forward rates need to be about 3 years apart in maturity for the size of the impact (from the same shock) on the volatility level to decrease by a half.

A totally different picture emerges from the LIFFE and TIFFE markets. We find that $\hat{\lambda}_{1}$ is not significantly different from zero, proving that these two markets do not price the jump risk. One may ask the question whether the absence of the jump risk pricing is the result of (i) the absence of a jump process in the market, ie. the market only experiences smooth changes in volatility, or (ii) the risk is negligible so that the market does not see the need to price it.

The value of $\gamma_{1}$ provides the answer to the above question. We point out that $\gamma_{1}$ can be interpreted as the average value of all jump sizes that come from the same jump noise, and therefore commands the same price of jump risk. Since $\hat{\gamma}_{1}$ is not 
significantly different from zero for the LIFFE market, one may infer that the jump component is absent in the U.K. market. On the other hand, the average jump size in the TIFFE market is statistically significantly different from zero. To reconcile with the result of no price of jump risk, it must be the case that the jump risk in this market is perceived as negligible, and so does not call for a price premium. At first glance, one might not be convinced that the market does not price a "significant" jump component. However, if we look at the magnitude of the jump components, the value of $\gamma_{1}$ during the period 1989-1994 was 0.0013, and during the period 1996-2001 was 0.000065 . These jump components may have been statistically significant, but they are not economically meaningful. It is reasonable that the market perceived them to be negligible.

Even though the absence of jump risk pricing in the U.K. and the Japanese markets is due to different reasons, what we should observe in reality is that both markets can be described by pure diffusion processes rather than jump-diffusion processes. Due to this non-identification of the null hypothesis (either $\gamma_{1}$ or $\lambda_{1}$ is equal to zero will result in no jump component), in Table 2 we did not report the likelihood ratio test for the TIFFE and LIFFE cases, and we did rely on the significance of estimated parameters in the jump-diffusion specification to draw conclusions about the markets.

Despite the fact that the instantaneous forward rate volatility function in the Japanese market has a humped-shape, we can see that the decay factor is very high, approximately 2.65 for the 2 periods, ie. the two forward rates need to be only about 3 months apart in maturity for the size of the impact (from the same diffusion shock) on the volatility level to decrease by a half. The impact of the shock on the long end of the yield curve, therefore, will be negligible. The instantaneous volatility for the sport rate is, however, higher than in other markets. It stays at $4.4 \%$ in the second period (1996-2001), which is double the $2 \%$ level in the first period (1989-1994). The second period is, therefore, characterized by a very low interest rate level $(0.5 \%)$ and high volatility.

6.2. Model validity check. We test for model validity by the score method. The score of the $j$ th observation is defined as the derivative of the natural logarithm of the conditional likelihood with respect to the parameter vector $\boldsymbol{\theta}$, namely

$$
\boldsymbol{h}_{j}(\boldsymbol{\theta})=\frac{\partial \ln p\left(\boldsymbol{G}_{j} \mid G_{j-1} ; \boldsymbol{\theta}\right)}{\partial \boldsymbol{\theta}} .
$$


If the model is correctly specified, the score $\boldsymbol{h}_{j}\left(\boldsymbol{\theta}_{0}\right)$ (evaluated at the true parameter value $\boldsymbol{\theta}_{0}$ ) should be impossible to forecast based on any past information, such as elements of the lagged score $\boldsymbol{h}_{j-k}\left(\boldsymbol{\theta}_{0}\right)(k \geq 1)$.

The test for serial correlation in the scores is proposed by White (1987), using the conditional moment tests of Newey (1985) and Tauchen (1985). Hamilton (1996) provides an excellent summary of the method, whose notation we use here.

First we collect in an $(l \times 1)$ vector $\boldsymbol{c}_{j}(\boldsymbol{\theta})$ those elements of the $H \times H$ matrix $\left[\boldsymbol{h}_{j}(\boldsymbol{\theta})\right]\left[\boldsymbol{h}_{j-1}(\boldsymbol{\theta})\right]^{\prime}$ that we want to confirm have a zero mean when evaluated at $\theta_{0}$. If the model is correctly specified, then

$$
\left[J^{-1 / 2} \sum_{j=1}^{J} \boldsymbol{c}_{t}(\hat{\boldsymbol{\theta}})\right]^{\prime} \cdot \widehat{\boldsymbol{A}}^{22} \cdot\left[J^{-1 / 2} \sum_{j=1}^{J} \boldsymbol{c}_{t}(\hat{\boldsymbol{\theta}})\right] \stackrel{d}{\rightarrow} \chi^{2}(l),
$$

where $\widehat{A}^{22}$ denotes the $(2,2)$ subblock of the inverse of the partitioned matrix

$$
\widehat{\boldsymbol{A}}=(1 / J) \cdot\left(\begin{array}{ll}
\sum_{j}\left[\boldsymbol{h}_{j}(\hat{\boldsymbol{\theta}})\right]\left[\boldsymbol{h}_{j}(\hat{\boldsymbol{\theta}})\right]^{\prime} & \sum_{j}\left[\boldsymbol{h}_{j}(\hat{\boldsymbol{\theta}})\right]\left[\boldsymbol{c}_{j}(\hat{\boldsymbol{\theta}})\right]^{\prime} \\
\sum_{j}\left[\boldsymbol{c}_{j}(\hat{\boldsymbol{\theta}})\right]\left[\boldsymbol{h}_{j}(\hat{\boldsymbol{\theta}})\right]^{\prime} & \sum_{j}\left[\boldsymbol{c}_{j}(\hat{\boldsymbol{\theta}})\right]\left[\boldsymbol{c}_{j}(\hat{\boldsymbol{\theta}})\right]^{\prime}
\end{array}\right)
$$

In our first test for the model's validity, we gathered all of the elements of the matrix $\left[\boldsymbol{h}_{j}(\boldsymbol{\theta})\right]\left[\boldsymbol{h}_{j-1}(\boldsymbol{\theta})\right]^{\prime}$ in the vector $\boldsymbol{c}_{j}(\boldsymbol{\theta})$. The test rejected the assumption of no serial correlation in the score in all of the cases. It is clear that the model proposed here is not good enough to capture the movements of the market.

To further investigate the mis-specification, we re-did the test for each element of the matrix $\left[\boldsymbol{h}_{j}(\boldsymbol{\theta})\right]\left[\boldsymbol{h}_{j-1}(\boldsymbol{\theta})\right]^{\prime}$ separately. The p-values for each test are reported in Table 3. The parameter set can be divided into two subsets, one consisting of $\sigma_{0}$, $\sigma_{1}$ and $\kappa$ which are associated with the Wiener noise, and the other consisting of $\kappa_{1}$, $\gamma_{1}$ and $\lambda_{1}$ which are associated with the Poisson noise. It can be seen that the serial correlation in the score mainly comes from the information content of the elements in each subset about themselves. Thus, the independence assumption between the Wiener and Poisson noise remains valid.

The score test clearly indicates the need for a better model specification. Some suggestions include (i) to incorporate level dependent diffusion volatility, (ii) to utilize a more elaborate jump volatility structure and (iii) to consider multi-factor specifications. We leave analysis of these suggestions for future research. 
TABLE 3. P-value for the score tests

This table reports the p-value for the score tests in the restricted models (ie. the best model for each exchange). For each test, the vector $\boldsymbol{c}_{j}(\boldsymbol{\theta})$ consists of only one element, which is the element $(i k)$ - row $i$, column $k$ - of the matrix $\left[\boldsymbol{h}_{j}(\boldsymbol{\theta})\right]\left[\boldsymbol{h}_{j-1}(\boldsymbol{\theta})\right]^{\prime}$. The p-value for that test is reported in the $(i k)$ position of the table.

(a) CME case

Humped diffusion volatility - Exponential jump volatility

\begin{tabular}{|l|lll|lll|l|}
\hline & \multicolumn{7}{|c|}{ Lagged variables } \\
\cline { 2 - 8 } & $\hat{\sigma}_{0}$ & $\hat{\sigma}_{1}$ & $\hat{\kappa}$ & $\hat{\kappa}_{1}$ & $\hat{\gamma}_{1}$ & $\hat{\lambda}_{1}$ & $\hat{\sigma}_{\epsilon}$ \\
\hline$\hat{\sigma}_{0}$ & 0.009 & 0.005 & 0.005 & 0.076 & 0.196 & 0.195 & 0.515 \\
$\hat{\sigma}_{1}$ & 0.016 & 0.002 & 0.003 & 0.043 & 0.217 & 0.217 & 0.113 \\
$\hat{\kappa}$ & 0.015 & 0.002 & 0.002 & 0.043 & 0.214 & 0.214 & 0.189 \\
\hline$\hat{\kappa}_{1}$ & 0.288 & 0.150 & 0.158 & 0.000 & 0.000 & 0.000 & 0.662 \\
$\hat{\gamma}_{1}$ & 0.401 & 0.266 & 0.253 & 0.000 & 0.000 & 0.000 & 0.654 \\
$\hat{\lambda}_{1}$ & 0.401 & 0.266 & 0.253 & 0.000 & 0.000 & 0.000 & 0.654 \\
\hline$\hat{\sigma}_{\epsilon}$ & 0.036 & 0.006 & 0.013 & 0.394 & 0.710 & 0.710 & 0.002 \\
\hline
\end{tabular}

(b) SFE case

Humped diffusion volatility - Constant jump volatility

\begin{tabular}{|l|lll|ll|l|}
\hline & \multicolumn{7}{|c|}{ Lagged variables } \\
\cline { 2 - 7 } & $\hat{\sigma}_{0}$ & $\hat{\sigma}_{1}$ & $\hat{\kappa}$ & $\hat{\gamma}_{1}$ & $\hat{\lambda}_{1}$ & $\hat{\sigma}_{\epsilon}$ \\
\hline$\hat{\sigma}_{0}$ & 0.005 & 0.116 & 0.712 & 0.030 & 0.030 & 0.016 \\
$\hat{\sigma}_{1}$ & 0.036 & 0.001 & 0.001 & 0.006 & 0.006 & 0.206 \\
$\hat{\kappa}$ & 0.307 & 0.001 & 0.001 & 0.032 & 0.032 & 0.723 \\
\hline$\hat{\gamma}_{1}$ & 0.237 & 0.474 & 0.764 & 0.029 & 0.029 & 0.653 \\
$\hat{\lambda}_{1}$ & 0.237 & 0.474 & 0.764 & 0.029 & 0.029 & 0.653 \\
\hline$\hat{\sigma}_{\epsilon}$ & 0.067 & 0.020 & 0.076 & 0.017 & 0.017 & 0.000 \\
\hline
\end{tabular}

(c) TIFFE case

Humped diffusion volatility - No jump component

\begin{tabular}{|c|cccc|cccc|}
\hline & \multicolumn{4}{|c|}{$1988-1994$} & \multicolumn{4}{c|}{$1996-2001$} \\
\cline { 2 - 9 } & $\hat{\sigma}_{0}$ & $\hat{\sigma}_{1}$ & $\hat{\kappa}$ & $\hat{\sigma}_{\epsilon}$ & $\hat{\sigma}_{0}$ & $\hat{\sigma}_{1}$ & $\hat{\kappa}$ & $\hat{\sigma}_{\epsilon}$ \\
\hline$\hat{\sigma}_{0}$ & 0.068 & 0.039 & 0.012 & 0.063 & 0.000 & 0.001 & 0.107 & 0.341 \\
$\hat{\sigma}_{1}$ & 0.048 & 0.022 & 0.008 & 0.026 & 0.001 & 0.005 & 0.096 & 0.150 \\
$\hat{\kappa}$ & 0.026 & 0.010 & 0.005 & 0.005 & 0.258 & 0.162 & 0.094 & 0.017 \\
$\hat{\sigma}_{\epsilon}$ & 0.084 & 0.035 & 0.007 & 0.027 & 0.291 & 0.134 & 0.022 & 0.001 \\
\hline
\end{tabular}

(d) LIFFE case

Constant diffusion volatility - No jump component

\begin{tabular}{|l|cc|}
\hline & lagged $\hat{\sigma}_{0}$ & lagged $\hat{\sigma}_{\epsilon}$ \\
\hline$\hat{\sigma}_{0}$ & 0.000 & 0.010 \\
$\hat{\sigma}_{\epsilon}$ & 0.225 & 0.200 \\
\hline
\end{tabular}




\section{CONClusion}

Under the framework of Heath, Jarrow and Morton (1992), we have proposed a generalized version of the Shirakawa (1991) model to capture the effect of jumps in the fixed income market. The model is based on an instantaneous forward rate specification, that allows the co-existence of a Wiener noise and multiple Poisson noises, each being associated with a time-deterministic volatility function. This specification with a finite number of jump components ensures the completeness of the market and the uniqueness of any contingent claim prices.

Based on this specification, we derive the evolution of futures prices, and prove that if the underlying instantaneous forward rate evolution contains a jump component, the jump must also present itself in the evolution of futures prices. This evolution can be used in the estimation process to determine the model parameters. The advantage of the approach is that we do not need to markovianize the system that contains the spot rate of interest or equivalently the bond prices, which may not always be possible. Instead, we treat the futures contract as a derivative instrument written on the instantaneous forward rate, and use the likelihood transformation method of Duan (1994) to estimate the model parameters.

The proposed approach is sufficiently general to deal with any specification with time deterministic diffusion and jump volatility functions. However, to illustrate our method, we choose a humped-shape specification for our diffusion volatility, and a "binomial" approach to model Poisson noise. We distinguish between information surprises that affect the whole yield curve more or less equally, and information surprises that are less prominent, and that affect some sections of the yield curve more than others. We run our estimation for 3-month futures contracts traded on the CME, SFE, LIFFE and TIFFE, the four large exchanges from different parts of the world.

The empirical results suggest very different jump behaviour in each market. The U.K market does not contain a jump component. The Japanese market has on average a positive jump size, and the jump risk must be perceived as negligible, since the market gives this jump risk zero price. On the other hand, a jump component is an important part of the U.S. and Australian markets. Given their very different characteristics, the mechanism by which a jump shock impacts the markets are also different. In the small Australian market, a shock has its impact spread out equally along the yield curve. In the U.S market, the impact of a shock dies out gradually along the yield 
curve, and therefore short term instruments are affected more heavily than longer term instruments.

However, model diagnostic tests indicate that the specification is still not rich enough to capture the true market behaviour. Some possible avenues to explore in order to improve the model fit include a level-dependent instantaneous forward rate volatility and extension to multi-factor noise terms. We intend to explore these issues in subsequent research.

\section{ApPENDiX A. Generalized Itô's LEMmA}

Consider a jump diffusion process in terms of the stochastic integral equation

$$
X_{t}=X_{0}+\int_{0}^{t} \alpha_{s} d s+\int_{0}^{t} \sigma_{s} d W_{s}+\int_{0}^{t} \delta_{s} d N_{s}
$$

or in terms of the stochastic differential equation

$$
d X=\alpha_{t} d t+\sigma_{t} d W(t)+\delta_{t} d N_{t}
$$

Given a $\mathcal{C}^{1,2}$-function $F(t, X)$, the stochastic differential equation for $F$ is

$$
\begin{aligned}
d F\left(t, X_{t}\right)= & {\left[F_{t}(\cdot)+F_{X}(\cdot) \alpha_{t} d t+\frac{1}{2} F_{X X}(\cdot) \sigma_{t}^{2}\right] d t } \\
& +F_{X} \sigma_{t} d W_{t}+\left[F\left(t, X_{t-}+\delta_{t}\right)-F\left(t, X_{t-}\right)\right] d N_{t}
\end{aligned}
$$

where

$$
\begin{aligned}
F_{t}(\cdot) & =\frac{\partial F\left(t, X_{t}\right)}{\partial t}, \\
F_{X}(\cdot) & =\frac{\partial F\left(t, X_{t}\right)}{\partial X}, \\
F_{X X}(\cdot) & =\frac{\partial^{2} F\left(t, X_{t}\right)}{\partial X^{2}} .
\end{aligned}
$$

APPENDIX B. The EXPECTATION OF A "LOG-POISSON" PROCESS

With a view to finding the second expectation in (3.1), we consider a variable $X$ whose evolution can be described by the stochastic integral equation

$$
X(T)=\int_{t}^{T} \zeta(u, \cdot) d N(u)
$$

where $N$ is a Poisson process with intensity $\lambda$. We are interested in the value $\mathbb{E}_{t}[\exp (X(T))]$. 
The value of $X(T)$ is just the sum of realized jump components, each appearing at the jump time $L_{i}$ between $t$ and $T$, ie.

$$
X(T)=\sum_{i=1}^{N(T)-N(t)} \zeta\left(L_{i}, \cdot\right)
$$

Using a standard result for conditional expectations we have that

$$
\mathbb{E}_{t}\left[\mathrm{e}^{X(T)}\right]=\sum_{n=0}^{\infty} \mathbb{E}_{t}\left[\mathrm{e}^{X(T)} \mid N(T)-N(t)=n\right] \mathbb{P}[N(T)-N(t)=n]
$$

First, consider the conditional expectation

$$
\mathbb{E}_{t}\left[\mathrm{e}^{X(T)} \mid N(T)-N(t)=n\right]=\mathbb{E}_{t}\left[\exp \left(\sum_{i=1}^{n} \zeta\left(L_{i}, \cdot\right)\right)\right]
$$

Lemma B.1. Given that $N(T)-N(t)=n$, the $n$ arrival times $L_{1}, L_{2}, \ldots, L_{n}$ have the same distribution as the order statistics corresponding to $n$ independent random variables uniformly distributed on the interval $(t, T)$.

Proof. See theorem 2.3.1 of Ross (1996), page 67.

The required conditional expectation thus becomes

$$
\begin{aligned}
& \mathbb{E}_{t}\left[\mathrm{e}^{X(T)} \mid(N(T)-N(t))=n\right]=\mathbb{E}_{t}\left[\exp \left(\sum_{i=1}^{n} \zeta\left(L_{i}, \cdot\right)\right)\right] \\
& =\left\{\mathbb{E}_{t}\left[\exp \left(\zeta\left(L_{i}, \cdot\right)\right)\right]\right\}^{n} \\
& =[\underbrace{\int_{t}^{T} \exp (\zeta(u, \cdot)) \frac{1}{T-t} d u}]^{n} \\
& \equiv \Psi^{n} \text {. }
\end{aligned}
$$


Substitute this result back to (B.1)

$$
\begin{aligned}
\mathbb{E}_{t}\left[\mathrm{e}^{X(T)}\right] & =\sum_{n=0}^{\infty} \Psi^{n} \mathbb{P}[N(T)-N(t)=n] \\
& =\sum_{n=0}^{\infty} \Psi^{n} \mathrm{e}^{-\lambda(T-t)} \frac{(\lambda(T-t))^{n}}{n !} \\
& =\mathrm{e}^{-\lambda(T-t)} \mathrm{e}^{\lambda(T-t) \Psi} \\
& =\exp \left\{\lambda \int_{t}^{T}[\exp (\zeta(u, \cdot))-1] d u\right\}
\end{aligned}
$$

\section{APPENDIX C. QUOTING CONVENTION OF EXCHANGES FOR FUTURES CONTRACTS}

Let $F_{j k} \equiv F\left(t, T_{F k}, T_{B k}\right)$ be the price at time $t$ of a futures contract maturing at time $T_{F k}(>t)$. The contract is written on a pure discount instrument which has a face value of $\$ 1$ and matures at time $T_{B k}\left(>T_{F k}\right)$.

We are considering 3-month futures contracts, and therefore $T_{B k}-T_{F k}=\tau$ constant for all $k \in[0, K]$.

The exchanges quote a value $G_{j k}$ which is linked with $F_{j k}$ via

$$
F_{j k}=\frac{1}{1+\left(1-\frac{G_{j k}}{N}\right) \tau} \equiv \eta\left(G_{j k}\right),
$$

where $\tau$ and $N$ vary across exchanges.

The CME and LIFFE have the same quoting convention, where $N=100$ and $\tau=$ $\frac{90}{360}$. The TIFFE's quoting convention was the same as the CME and LIFFE for the period prior to October 1,1999 , after which date $N=1000$ has been used. The SFE also takes $N=100$, but uses 365-day-year basis, ie. $\tau=\frac{90}{365}$.

\section{APPENDIX D. FULL INFORMATION LOG LIKELIHOOD FUNCTION FOR QUOTED FUTURES PRICES}

The main task in deriving the log likelihood function is to calculate the Jacobian of the transformation and write out the drift vector and covariance matrix for each transition log likelihood function. These quantities then can be substituted directly to 
the likelihood formulae in the text (equations 4.7, 4.10, 4.11 and 4.12) to write out the likelihood function for observable futures prices.

From (4.3)

$$
X_{j k}=\ln \left(F_{j k}\right) \equiv \zeta\left(F_{j k}\right)
$$

we have

$$
\frac{\partial \zeta\left(F_{j k} ; \boldsymbol{\theta}\right)}{\partial F_{j k}}=\frac{1}{F_{j k}}
$$

From (4.2)

$$
F_{j k}=\frac{1}{1+\left(1-\frac{G_{j k}}{100}\right) \tau} \equiv \eta\left(G_{j k}\right),
$$

where $\tau=90 / 360$ for CME Eurodollar futures, we find that

$$
\frac{\partial \eta\left(G_{j k} ; \theta\right)}{\partial G_{j k}}=\frac{-\frac{\tau}{100}}{\left[1+\left(1-\frac{G_{j k}}{100}\right) \tau\right]^{2}} .
$$

The calculations for other exchanges are very similar, and therefore will be omitted here.

The variance $(4.5)$ is

$$
\begin{aligned}
b_{j(k k)}= & \int_{t_{j-1}}^{t_{j}}\left(\beta_{k}^{2}(u)+\sigma_{\varepsilon}^{2}\right) d u \\
= & \int_{t_{j-1}}^{t_{j}}\left(\int_{T_{F_{k}}}^{T_{B_{k}}} \sigma(u, s) d s\right)^{2} d u+\int_{t_{j-1}}^{t_{j}} \sigma_{\varepsilon}^{2} d u \\
= & M^{2} I_{00}+2 M N I_{01}+N^{2} I_{02}+2 M R I_{11} \\
& +2 N R I_{12}+R^{2} I_{22}+\sigma_{\varepsilon}^{2}\left(t_{j}-t_{j-1}\right),
\end{aligned}
$$


where

$$
\begin{aligned}
M & =\sigma_{0}\left(T_{B k}-T_{F k}\right) \\
N & =-\left(\frac{\sigma_{0}}{\kappa}+\frac{\sigma_{1}}{\kappa^{2}}\right)\left(\mathrm{e}^{-\kappa T_{B k}}-\mathrm{e}^{-\kappa T_{F k}}\right)-\frac{\sigma_{1}}{\kappa}\left(T_{B k} \mathrm{e}^{-\kappa T_{B k}}-T_{F k} \mathrm{e}^{-\kappa T_{F k}}\right), \\
R & =\frac{\sigma_{1}}{\kappa}\left(\mathrm{e}^{-\kappa T_{B k}}-\mathrm{e}^{-\kappa T_{F k}}\right) \\
I_{a b} & =\int_{t_{j-1}}^{t_{j}} \tau^{a} \mathrm{e}^{\kappa b \tau} d \tau \\
= & \left(-\mathrm{e}^{\kappa b \tau}\left[\frac{1}{(-\kappa b)} \tau^{a}+\frac{a}{(-\kappa b)^{2}} \tau^{a-1}+\frac{a(a-1)}{(-\kappa b)^{3}} \tau^{a-2}+\ldots\right.\right. \\
& \left.\left.\ldots+\frac{a(a-1) \ldots 2}{(-\kappa b)^{a}} \tau+\frac{a(a-1) \ldots 1}{(-\kappa b)^{a+1}} \tau^{0}\right]\right)\left.\right|_{t_{j-1}} ^{t_{j}}
\end{aligned}
$$

(use successive integration by part).

The covariance (4.6) is

$$
\begin{aligned}
b_{j\left(k_{1} k_{2}\right)}= & \int_{t_{j-1}}^{t_{j}} \beta_{k_{1}}(u) \beta_{k_{2}}(u) d u \quad\left(\text { for } k_{1} \neq k_{2}\right) \\
= & \int_{t_{j-1}}^{t_{j}}\left(\int_{T_{F k_{1}}}^{T_{B k_{1}}} \sigma(u, s) d s\right)\left(\int_{T_{F k_{2}}}^{T_{B k_{2}}} \sigma(u, s) d s\right) d u \\
= & M_{1} M_{2} I_{00}+\left(M_{1} N_{2}+N_{1} M_{2}\right) I_{01}+N_{1} N_{2} I_{02} \\
& +\left(M_{1} R_{2}+R_{1} M_{2}\right) I_{11}+\left(N_{1} R_{2}+R_{1} N_{2}\right) I_{12}+R_{1} R_{2} I_{22},
\end{aligned}
$$

where

$$
\begin{aligned}
& M_{1}=\sigma_{0}\left(T_{B k_{1}}-T_{F k_{1}}\right), \\
& M_{2}=\sigma_{0}\left(T_{B k_{2}}-T_{F k_{2}}\right), \\
& N_{1}=-\left(\frac{\sigma_{0}}{\kappa}+\frac{\sigma_{1}}{\kappa^{2}}\right)\left(\mathrm{e}^{-\kappa T_{B k_{1}}}-\mathrm{e}^{-\kappa T_{F k_{1}}}\right)-\frac{\sigma_{1}}{\kappa}\left(T_{B k_{1}} \mathrm{e}^{-\kappa T_{B k_{1}}}-T_{F k_{1}} \mathrm{e}^{-\kappa T_{F k_{1}}}\right), \\
& N_{2}=-\left(\frac{\sigma_{0}}{\kappa}+\frac{\sigma_{1}}{\kappa^{2}}\right)\left(\mathrm{e}^{-\kappa T_{B k_{2}}}-\mathrm{e}^{-\kappa T_{F k_{2}}}\right)-\frac{\sigma_{1}}{\kappa}\left(T_{B k_{2}} \mathrm{e}^{-\kappa T_{B k_{2}}}-T_{F k_{2}} \mathrm{e}^{-\kappa T_{F k_{2}}}\right), \\
& R_{1}=\frac{\sigma_{1}}{\kappa}\left(\mathrm{e}^{-\kappa T_{B k_{1}}}-\mathrm{e}^{-\kappa T_{F k_{1}}}\right), \\
& R_{2}=\frac{\sigma_{1}}{\kappa}\left(\mathrm{e}^{-\kappa T_{B k_{2}}}-\mathrm{e}^{-\kappa T_{F k_{2}}}\right),
\end{aligned}
$$

and $I_{a b}$ are defined as in (D.1). 
The mean term (4.8) is

$$
\begin{aligned}
a_{j k(0)}= & \int_{t_{j-1}}^{t_{j}}\left(\mu_{k}(u)-\frac{1}{2} \sigma_{\varepsilon}^{2}\right) d u \\
= & -\frac{1}{2} b_{j(k k)}-\sum_{m=1}^{M} \lambda_{m} \int_{t_{j-1}}^{t_{j}}\left[\exp \left(-\int_{T_{F k}}^{T_{B k}} \delta_{m}(u, s) \gamma_{m} d s\right)-1\right] d u \\
= & -\frac{1}{2} b_{j(k k)}+\sum_{m=1}^{M} \lambda_{m}\left(t_{j}-t_{j-1}\right) \\
& -\frac{\lambda_{1}}{\kappa_{1}}\left(E_{1}\left(-c_{k} \mathrm{e}^{-\kappa_{1} t_{j-1}},-c_{k} \mathrm{e}^{-\kappa_{1} t_{j}}\right)\right) \\
& -\lambda_{2}\left[\exp \left(-\gamma_{2}\left(T_{B k}-T_{F k}\right)\right)-1\right]\left(t_{j}-t_{j-1}\right)
\end{aligned}
$$

where

$$
c_{k}=\frac{\gamma_{1}}{\kappa_{1}}\left(\mathrm{e}^{-\kappa_{1} T_{B_{k}}}-\mathrm{e}^{-\kappa_{1} T_{F_{k}}}\right),
$$

and $E_{1}\left(z_{1}, z_{2}\right)$ is the exponential integral ${ }^{13}$ defined by

$$
E_{1}\left(z_{1}, z_{2}\right)=\int_{z_{1}}^{z_{2}} \frac{\mathrm{e}^{-u}}{u} d u, \quad\left(z_{1} z_{2}>0\right)
$$

The mean term (4.9) is

$$
a_{j k(m)}=\int_{t_{j-1}}^{t_{j}}\left(\mu_{k}(u)-\frac{1}{2} \sigma_{\varepsilon}^{2}+\nu_{m k}(u)\right) d u \quad(\text { for } m \neq 0) .
$$

Evaluated the integration gives

$$
\begin{aligned}
& a_{j k(1)}=a_{j k(0)}+c_{k} \frac{\mathrm{e}^{\kappa_{1} t_{j}}-\mathrm{e}^{\kappa_{1} t_{j-1}}}{\kappa_{1}}, \\
& a_{j k(2)}=a_{j k(0)}-\gamma_{m}\left(T_{B k}-T_{F k}\right)\left(t_{j}-t_{j-1}\right) .
\end{aligned}
$$

\section{REFERENCES}

Abramowitz, M. and Stegun, I. A. (1965), Handbook of Mathematical Functions - With Formulas, Graphs, and Mathematical Tables, Dover Publications Inc.

Amin, K. I. and Morton, A. J. (1994), 'Implied Volatility Functions in Arbitrage-free Term Structure Models', Journal of Financial Economics 35, 141-180.

Babbs, S. and Webber, N. (1995), A Theory of the Term Structure with an Official Short Rate, Working Paper, University of Warwick.

\footnotetext{
${ }^{13}$ The exponential integral evaluation is available from most numerical packages, such as Ox, Matlab and Mathematica. An approximation formula can be found in Abramowitz and Stegun (1965).
} 
Balduzzi, P., Green, T. and Elton, E. (1998), Economic News and the Yield Curve: Evidence from the U.S. Treasury Market, Working Paper, New York University.

Ball, C. A. and Torous, W. N. (1983), 'A Simplified Jump Process for Common Stock Returns', Journal of Financial and Quantitative Analysis 18(1), 53-65.

Ball, C. A. and Torous, W. N. (1985), 'On Jumps in Common Stock Prices and Their Impact on Call Option Pricing', Journal of Finance 40(1), 155-173.

Bates, D. (1996), 'Jumps and Stochastic Volatility: Exchange Rate Processes Implicit in DM Options', Review of Financial Studies 9(1), 69-107.

Bates, D. (2000), 'Post-87 Crash fears in the S\&P 500 Futures Option Market', Journal of Econometrics 94, 181-238.

Bhar, R., Chiarella, C. and Tô, T.-D. (2002), A Maximum Likelihood Approach to Estimation of a Class of the Heath-Jarrow-Morton Models, Working Paper No 80, Quantitative Finance Research Group, University of Technology, Sydney.

Björk, T., Kabanov, Y. and Runggaldier, W. (1997), 'Bond Market Structure in the Presence of Marked Point Processes', Mathematical Finance 7(2), 211-223.

Björk, T. and Landén, C. (2002), 'On the Term Structure of Futures and Forward Prices', pp. 111-150. in Mathematical Finance - Bachelier Congress 2000, ed. G. Helyette, D. Madan, S. Pliska, T. Vorst, Springer-Verlag.

Chacko, G. (1998), Continuous Time Estimation of Exponential Separable Term Structure Models: A General Approach, Working Paper, Harvard Business School.

Chacko, G. and Das, S. (2002), 'Pricing Interest Rate Derivatives: A General Approach', Review of Financial Studies 15(1), 195-241.

Chiarella, C. and Kwon, O. (2002b), Finite Dimensional Realisations of Interest Rate Models Driven by Marked Point Processes, Working Paper, Quantitative Finance Research Group, University of Technology, Sydney.

Chiarella, C. and Nikitopoulos Sklibosios, C. (2002), A Jump-Diffusion Derivative Pricing Model Arising Within the Heath-Jarrow-Morton Framework, Working paper, Quantitative Finance Research Group, University of Technology, Sydney.

Cox, J. C., Ingersoll, J. and Ross, S. A. (1981), 'The Relationship Between Forward Prices and Futures Prices', Journal of Financial Economics 9, 321-346.

Das, S. R. (2002), 'The Surprise Element: Jumps in Interest Rates', Journal of Econometrics 106, 27 65.

Doornik, J. A. (1996), Ox: An Object-Oriented Matrix Programming Language, International Thomson Business Press.

Duan, J.-C. (1994), 'Maximum Likelihood Estimation Using Price Data of the Derivative Contract', Mathematical Finance 4(2), 155-167. Duan (2000) 'Correction: Maximum Likelihood Estimation Using Price Data of the Derivative Contract' Mathematical Finance, 10(4), 461-462.

Dwyer, G. and Hafer, H. W. (1989), Interest Rates and Economic Announcements, Technical report, Federal Reserve Bank of St.Louis. 
El-Jahel, L., Lindberg, H. and Perraudin, W. (1997), Interest Rate Distributions, Yield Curve Modelling and Monetary Policy, in M. A. H. Dempster and S. R. Pliska, eds, 'Mathematics of Derivative Securities', Cambridge University Press.

Green, T. C. (1998), News Releases, Asymmetric Information, and Intraday Price Formation in the Interdealer Market for Treasury Securities, Working Paper, New York University.

Hamilton, J. D. (1994), Time Series Analysis, Princeton University Press, Princeton, New Jersey.

Hamilton, J. D. (1996), 'Specification Testing in Markov-Switching Time-Series Models', Journal of Econometrics 70, 127-157.

Hardouvelis, G. A. (1988), 'Economic News, Exchange Rates and Interest Rates', Journal of International Money and Finance 7(1), 23-35.

Heath, D., Jarrow, R. and Morton, A. (1992), 'Bond Pricing and the Term Structure of Interest Rates: A New Methodology for Contingent Claims Valuation', Econometrica 60(1), 77-105.

Ho, T. S. Y. and Lee, S. B. (1986), 'Term Structure Movements and Pricing Interest Rate Contingent Claims', Journal of Finance 41, 1011-1029.

Honoré, P. (1998), 'Pitfalls in Estimating Jump-Diffusion Models'. Working paper series No18, Centre for Analytical Finance, University of Aarhus.

Hull, J. and White, A. (1990), 'Pricing Interest-Rate-Derivative Securities', Review of Financial Studies 3(4), 573-592.

Jiang, G. J. (1999), 'Jump-Diffusion Model of Exchange Rate Dynamics - Estimation via Indirect Inference', Issues in Computational Economics and Finance . edited by S. Holly and S. Greenblatt, Amsterdam: Elsevier.

Jorion, P. (1988), 'On Jump Processes in the Foreign Exchange and Stock Markets', The Review of Financial Studies 1(4), 427-445.

Kiefer, N. M. (1978), 'Discrete Parameter Variation: Efficient Estimation of a Switching Regression Model', Econometrica 46(2), 427-434.

Lo, A. W. (1988), 'Maximum Likelihood Estimation of Generalized Itô Processes with Discretely Sampled Data', Econometric Theory 4(2), 231-247.

Merton, R. C. (1976), 'Option Pricing When Underlying Stock Returns Are Discontinuous', Journal of Financial Economics 3, 125-144.

Musiela, M., Turnbull, S. M. and Wakeman, L. M. (1992), 'Interest Rate Risk Management', Review of Futures Market 12(2), 221-261.

Naik, V. and Lee, M. H. (1995), Yield Curve Dynamics with Discrete Shifts in Economic Regimes: Theory and Estimation, Working Paper, University of Bristish Columbia.

Newey, W. K. (1985), 'Maximum Likelihood Specification Testing and Conditional Moment Tests', Econometrica 53, 1047-1070.

Pan, J. (2002), 'The Jump-Risk Premia Implicit in Options: Evidence from an Integrated Time-Series Study', Journal of Financial Economics 63, 3-50.

Piazzesi, M. (1999), An Econometric Model of the Yield Curve with Macroeconomic Jump Effects, Working Paper Number 8246, NBER. 
Quandt, R. E. and Ramsey, J. B. (1978), 'Estimating Mixtures of Normal Distributions and Switching Regressions', Journal of the American Statistical Association 73(361), 730-738. Applications Section.

Raj, M., Sim, A.-B. and Thurston, D. C. (1997), 'A Generalized Method of Moments Comparison of the Cox-Ingersoll-Ross and Heath-Jarrow-Morton Models', Journal of Economics and Business 49(2), 169-192.

Ross, S. M. (1996), Stochastic Processes, 2nd edn, John Wiley and Sons, Inc.

Shirakawa, H. (1991), 'Interest Rate Option Pricing with Poisson-Gaussian Forward Rate Curve Processes', Mathematical Finance 1(4), 77-94.

Singleton, K. (2001), 'Estimation of Affine Asset Pricing Models Using the Empirical Characteristic Function', Journal of Econometrics 102(1), 111-141.

Tauchen, G. (1985), 'Diagnostic Testing and Evaluation of Maximum Likelihood Models', Journal of Econometrics 30, 415-443.

White, H. (1987), Specification Testing in Dynamic Models, in T. F. Bewley, ed., 'Advances in Econometrics', Vol. 2, Cambridge University Press, Cambridge. Fifth world congress. 